\title{
Sino-InSpace: A Digital Simulation Platform for Virtual Space Environments
}

\author{
Liang Lyu ${ }^{1,2} \oplus$, Qing $X u^{1,3}$, Chaozhen Lan ${ }^{1, *}$, Qunshan Shi ${ }^{1}$, Wanjie $\mathrm{Lu}^{1,2}$, Yang Zhou ${ }^{1}$ and \\ Yinghao Zhao ${ }^{1,2}$ \\ 1 Zhengzhou Institute of Surveying and Mapping, Zhengzhou 450052, China; lvliangvip@163.com (L.L.); \\ 13937169139@139.com (Q.X.); hills1@163.com (Q.S.); lwj285149763@163.com (W.L.); \\ zhouyang3d@163.com (Y.Z.); zyh_message@163.com (Y.Z.) \\ 2 Science and Technology on Information System Engineering Laboratory, Nanjing Research Institute of \\ Electronics Engineering, Nanjing 210007, China \\ 3 Collaborative Innovation Center of Geo-Information Technology for Smart Central Plains, \\ Zhengzhou 450001, China \\ * Correspondence: 13014511234@163.com; Tel.: +86-130-1451-1234
}

Received: 14 July 2018; Accepted: 3 September 2018; Published: 8 September 2018

\begin{abstract}
The implementation of increased space exploration missions reduces the distance between human beings and outer space. Although it is impossible for everyone to enter the remote outer space, virtual environments could provide computer-based digital spaces that we can observe, participate in, and experience. In this study, Sino-InSpace, a digital simulation platform, was developed to support the construction of virtual space environments. The input data are divided into two types, the environment element and the entity object, that are then supported by the unified time-space datum. The platform adopted the pyramid model and octree index to preprocess the geographic and space environment data, which ensured the efficiency of data loading and browsing. To describe objects perfectly, they were abstracted and modeled based on four aspects including attributes, ephemeris, geometry, and behavior. Then, the platform performed the organization of a visual scenario based on logical modeling and data modeling; in addition, it ensured smooth and flexible visual scenario displays using efficient data and rendering engines. Multilevel modes (application directly, visualization development, and scientific analysis) were designed to support multilevel applications for users from different grades and fields. Each mode provided representative case studies, which also demonstrated the capabilities of the platform for data integration, visualization, process deduction, and auxiliary analysis. Finally, a user study with human participants was conducted from multiple views (usability, user acceptance, presence, and software design). The results indicate that Sino-InSpace performs well in simulation for virtual space environments, while a virtual reality setup is beneficial for promoting the experience.
\end{abstract}

Keywords: virtual space environments; Sino-InSpace; simulation platform; visualization scenario; multilevel application; user evaluation

\section{Introduction}

The astounding advances in aerospace technology are making the dream of space exploration come true. Since the launch of the first artificial satellite by the Soviet Union in 1957, human beings have been constantly exploring the vastness of the universe, which includes the near-earth space [1], the moon, Mars, and asteroids [2]. Chinese space activities started with the launch of Dong Fang Hong I in 1970; since then, a number of BeiDou navigation satellites, resource remote sensing satellites, and Fēngyún meteorological satellites had been sent into operation. In addition, China's progress in 
deep space exploration is also noteworthy [3]. The Chang'E lunar exploration program, which involves achieving lunar orbit, soft-landing on the moon, and returning a lunar sample has now entered its third stage [4]. The first Chinese Mars exploration mission has also been formally approved, and a Mars probe is planned for launch in the second half of 2020. With the possible opportunities and new avenues that space exploration promises, especially considering the problems of environmental pollution and resource depletion, many countries have set up ambitious space programs [5-7]. For human beings, outer space is a relatively "new world" and difficult to reach currently. However, virtual environments, initially viewed as "mirror worlds", can supply computer-based digital spaces that we observe, participate in, and experience in person [8]. With the increasing scope and scale of human space activities, it is essential to construct a digital simulation platform for virtual space environments (VSEs).

The traditional geographical information disciplines primarily study the Earth's surface including commercial (e.g., ArcGIS, MapInfo, and SuperMap) or open source geographic information system (GIS) platforms [9]; these platforms focus on processing, expressing, and analyzing the surface geographical data that are closely related to daily production and life. With the data acquired by deep space probes, the visualization of the planets' surface map based on GIS has been studied by some organizations such as the 'Multilingual Maps of the Terrestrial Planets and their Moons' [10], 'Lunar Web Mapping Application' [11], 'Mars Global GIS Mapping Application' [12], and Gazetteer of Planetary Nomenclature [13]; however, these maps are often relatively simple and lack effective three-dimensional (3D) visualization. Since the concept of a digital globe was proposed in 1998 by U.S. Vice President Al Gore, a large number of platforms such as Google Earth [14], World Wind [15], ArcGIS Earth [16], DEPS/CAS [17], and GeoGlobe [18] have been developed, providing a new means to understand the Earth. Furthermore, some similar digital visualization platforms such as Moonworld [19], Sino-VirtualMoon [20], Google Mars [21], JMARS [22], and ADVISER [23,24] have been developed, which can intuitively display the images of planetary surfaces and depict information regarding their topography, geology, and landing point through virtual reality (VR) while also supporting collaborative simulation. However, these platforms are often limited to a single planet and lack the capability to simulate the entire process of complex space-based activities. Due to the specialization of space science, there are not many mature aerospace mission simulation platforms thus far. Systems Tool Kit (STK) [25], developed by AGI, is a leading commercial software used for analysis in the aerospace field, which can support space exploration simulation including design, testing, launch, operation, and task application. Recently, the company also introduced a simplified open-source, web-based version of the platform called Cesium [26]. In addition, World Wind, General Mission Analysis Tool (GMAT) [27], SaVi [28], 3DView [29], and Cosmographia [30] can offer certain capabilities for track design, simulation, and scientific data visualization; moreover, these were made open source for the public. These aforementioned platforms focus on space activity simulation and have inadequate features for the analysis and visualization of a planet's surface information. In addition, these platforms require professional knowledge to operate, and would not be widely accepted by the general public such as the case of Google Earth [31]. The virtual geographic environments (VGEs) aim to provide integrated tools to understand the world better and have been widely used in multiple fields, such as air pollution experiments, silt dam planning, and group behavior simulation [8,32-39]. In fact, VSEs can be regarded as the extension of VGEs in the field of outer space, and some ideas derived from VGEs can also be used for reference.

A digital simulation platform called Sino-InSpace was developed for the abstract representations, visualizations with multiple viewpoints, and analytical understanding of VSEs from Earth to the outer planets. Using this platform, space activities and the access and analysis of geological information can be supported. In addition, it can provide simulation technology for the development of VGEs, the improvement of public universe cognition, and space science exploration. The remainder of the paper is organized as follows. The orientation and data source of Sino-InSpace are analyzed in Section 2. Section 3 illustrates the key technologies including time-space datum, data organization 
and preprocessing, visualization scenario organization, and design of the visualization engine. Three types of application modes were designed, and related case studies are introduced in Section 4 . Then, a user study with participants was conducted to evaluate the performance of the platform in Section 5 . Finally, we discuss the features and future work of the platform in Section 6.

\section{Platform Orientation and Data Source Analysis}

Compared to the majority of the digital earth and VGE platforms, Sino-InSpace focuses on visualization and analysis on a larger scale space including the construction of the virtual interplanetary space environment, digital planets, space environment/weather monitoring, space mission launch, space objects collision warning, landing area selection, and so on (Figure 1).

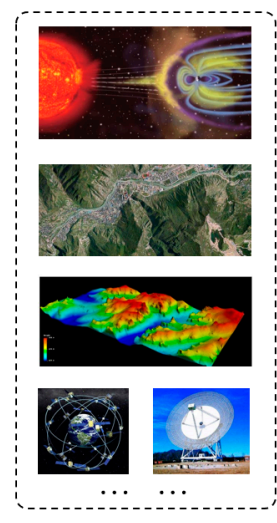

Data

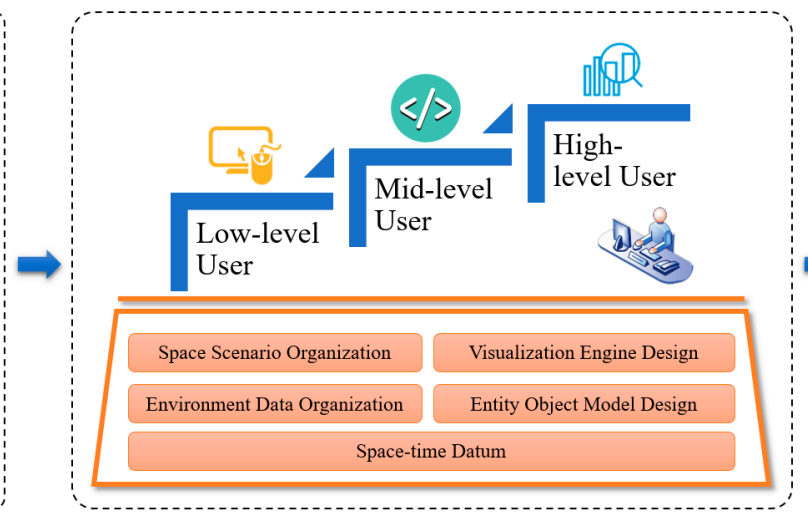

Architecture of Sino-InSpace

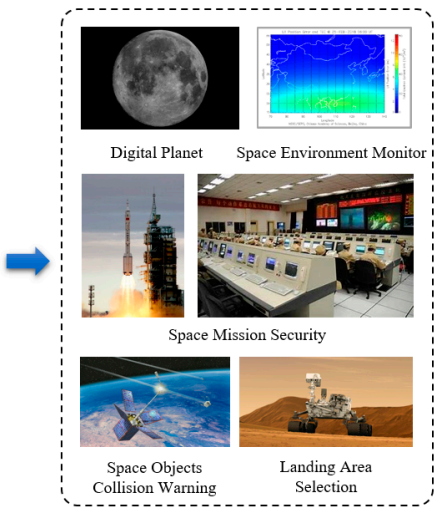

Application

Figure 1. Platform orientation.

Complete VGEs can be divided into four subtypes: the data environment, modeling environment, expression environment, and collaborative environment $[8,35,40,41]$. The data environment is fundamental for the other three subtypes [42]. Therefore, the data source of VSEs is analyzed first and divided into two types: the environment element and the entity object element (Table 1).

Table 1. Data source of virtual space environments.

\begin{tabular}{|c|c|c|}
\hline \multicolumn{2}{|c|}{ Data Type } & Content \\
\hline \multirow[b]{2}{*}{$\begin{array}{l}\text { Environment } \\
\text { element }\end{array}$} & $\begin{array}{l}\text { Basic geographic } \\
\text { environment }\end{array}$ & $\begin{array}{l}\text { Remote sensing images on the surface of the planet, digital } \\
\text { elevation model (DEM), and vector and feature data. }\end{array}$ \\
\hline & $\begin{array}{l}\text { Physical space } \\
\text { environment/weather }\end{array}$ & $\begin{array}{l}\text { The position, distribution, temperature and intensity information } \\
\text { of physical space environment such as middle and upper } \\
\text { atmosphere, geomagnetic field, ionosphere, radiant zone, and so } \\
\text { on. The track and size information of space debris. }\end{array}$ \\
\hline \multirow{4}{*}{$\begin{array}{l}\text { Entity object } \\
\text { element }\end{array}$} & Background star & $\begin{array}{c}\text { Astronomical catalogues of stars containing accurate position, } \\
\text { proper motion, level, and color. }\end{array}$ \\
\hline & $\begin{array}{l}\text { Planet in the solar } \\
\text { system }\end{array}$ & $\begin{array}{l}\text { The size, shape, texture, rotation speed, etc. of planets. } \\
\text { Astronomical ephemerides used to calculate the position and } \\
\text { velocity of planets. }\end{array}$ \\
\hline & Spacecraft & $\begin{array}{l}\text { Orbit elements, trajectories, attitudes, 3D models, and attributes of } \\
\text { artificial satellites and spacecraft. }\end{array}$ \\
\hline & Ground facility & $\begin{array}{l}\text { The location, model, capability, and attribute data of all kinds of } \\
\text { ground facilities such as optical telescopes, radar, launching and } \\
\text { measurement, and control stations. }\end{array}$ \\
\hline
\end{tabular}

The environment elements are the basis for building the virtual space. In addition to the image, digital elevation model (DEM) and other basic geographic data, space activities also need to consider 
the space environment such as the atmosphere, magnetic field, ionosphere, and space debris around the spacecraft during the flight. With all kinds of earth observation systems, we have accumulated considerable geographical environment data. For the Moon, Mars, asteroids, and other celestial bodies, we can also obtain rich remote sensing images and topographic data through detectors, and these data enhance the cognition of the planets. The data source of the space environment includes two types: (i) data collected by sensing instruments $[43,44]$ and (ii) data calculated by empirical models (e.g., NRLMSISE-00 empirical model [45], IGRF-12 model [46], and IRI2012 model [47]) developed using sensory data. With these data sources, datasets such as the position distribution, intensity, and density of various elements are achieved.

Entity objects refer to the targets of direct or indirect participation in space activities, and related elements include the following types. (i) Background star. Sino-InSpace adopts the Tycho-2 catalogue [48] to calculate the current position, brightness, and size of stars, which are then described using the point-based rendering. (ii) Planet in the solar system. The data includes the radius, eccentricity, texture, rotation speed, etc. The speed and position are calculated by deploying the NASA Jet Propulsion Laboratory Development Ephemeris (JPL DE) 405 [49]. (iii) Spacecraft. As the main participants of space activities, the platform focuses on the expression of its position, posture, form, and attribute information. (iv) Ground facility. They are usually used as auxiliary parts of space activities including observation equipment, data transmission and receiving equipment, launch sites, and so forth. Compared with environment elements, entity object elements focus on the extraction of overall information, e.g., location, form, capability, etc.

The storage and management of the above data can be carried out in a variety of ways such as files, databases, network services, etc., which are not the focus of the platform construction. To build VSEs, the platform mainly solves the problems of data organization, target modeling, scene organization, and visualization under the unified time-space datum. In addition, taking into account the needs of low-, medium-, and high-level users, three application modes (application directly, visualization development, and scientific analysis) were designed correspondingly. The difficulty of the application mode increased gradually, while the activity that can be simulated will be more complex. Taking teachers who are engaged in popular science education of the moon as an example, they may be considered as low-level users and always do some simple work in the application directly mode. In this case, the platform is used to display the shape, topography, and famous craters of the planet directly without any development. Accordingly, medium- and high-level users usually have further needs regarding application and research, such as individualized customization, real-time data monitoring, analysis of a space event, etc. Current functions of the platform are not enough, so these demands can be realized based on the reserved interfaces and modules in the other two modes.

\section{Key Technologies}

The data source elements that Sino-InSpace can accommodate increases considerably in category, quantity, and scale when compared with most of the current simulation platforms for the earth space. Therefore, based on a unified space-time datum, Sino-InSpace needs proper strategies to satisfy the requirements of visualization representation and analysis. It should be noted that Sino-InSpace was developed using the programming languages $\mathrm{C}++$ and $\mathrm{Qt}$, while the rendering engine was realized on an OpenGL 3.0 platform independently. Consequently, the cross-platform features were ensured.

\subsection{Time-Space Datum}

Time and space domains are basic attributes of an object. Thus, accurate space-time datum is a prerequisite to depict and cognize an object; these datums are maintained by many specialized research institutes [50-52]. Due to the different sources of data and means of obtaining these data in practical applications, the spatial-temporal coordinate format of the data and model interfaces vary widely. Therefore, it is extremely essential for Sino-InSpace to clarify the time-space reference and perform mutual conversions. 
From observations of the sun to stars, and then to the introduction of the International Atomic Time (TAI) in 1967, the development of the time system has been guided by the pursuit of higher accuracy. The current time system primarily consists of Sidereal Time (ST), Universal Time (UT), Ephemeris Time (ET), Dynamical Time (DT), and Atomic Time. In addition, other time references have been defined, such as Coordinated Universal Time (UTC), which has been adopted within the Sino-InSpace, Global Positioning System Time (GPST), and BeiDou Navigation Satellite System Time (BDT) for convenience. The conversion between time systems when the data are imported or exported is accomplished with the help of the Standards of Fundamental Astronomy (SOFA) Library for ANSI C [53].

Considering the space datum, the primary scope of human activity is currently in the earth circle; therefore, we considered a coordinate reference system centered on the earth. Nevertheless, the activities at the solar system scale needed to be supported by a coordinate system with a larger scale. These large-scale coordinate systems [54-56] primarily included the International Celestial Reference System (ICRS), Planetary Centroid Reference System (PCRS), International Terrestrial Reference System (ITRS), Station Coordinate System, and Satellite Coordinate System, as shown in Figure 2.

In addition, the transitional coordinate system was used to meet the needs of coordinate system conversion. For example, the orbit prediction of an Earth-center satellite is mostly carried out in the J2000 coordinate system (there is a small deviation matrix between it and the Geocentric Celestial Reference System (GCRS)). However, substellar point calculation and coverage analysis are often carried out in ITRS. Therefore, the transformation between these systems requires the celestial intermediate reference system and terrestrial intermediate reference system. The conversion between reference systems in Sino-InSpace is also supported by the SOFA Library [53].

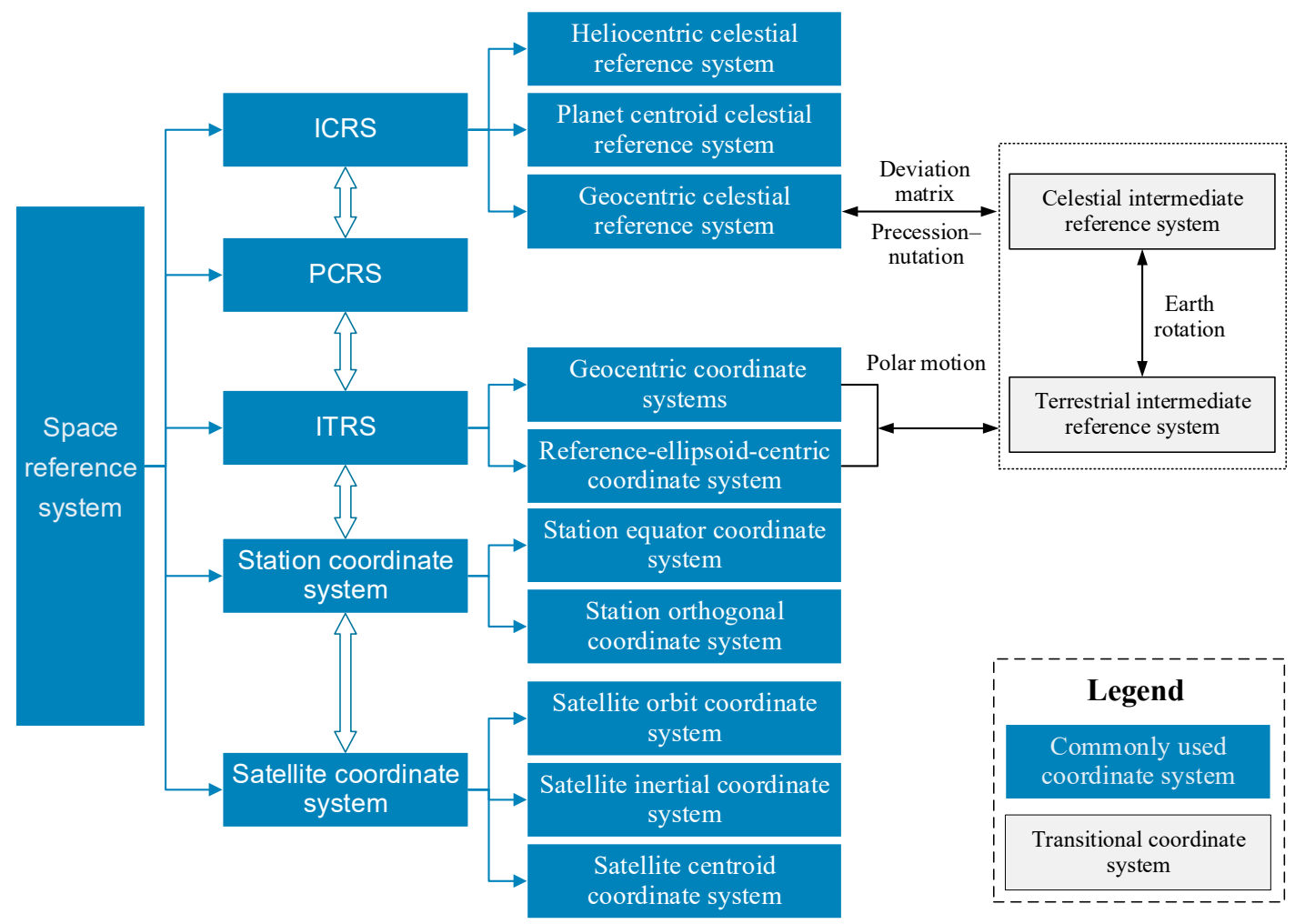

Figure 2. Space datum of the Sino-InSpace platform. 


\subsection{Environment Data Organization}

\subsubsection{Organization of Geographical Environment Data}

Geographical environment data is the basis of human activities on the surface of a planet, which includes all kinds of data collected by probes, primarily remote images of the planet surface, DEMs, vectors, and toponymic data, among others. To improve the efficiency of data loading and rendering, the strategy of the multiresolution pyramid model is generally adopted by most digital planet systems [57,58] depending on a structure known as a discrete global grid system (DGGS) [59]. The traditional approach discretizing a solid planet is to use the latitude/longitude coordinate system, which was also adopted in the early stages of the platform development (see Figure 3).
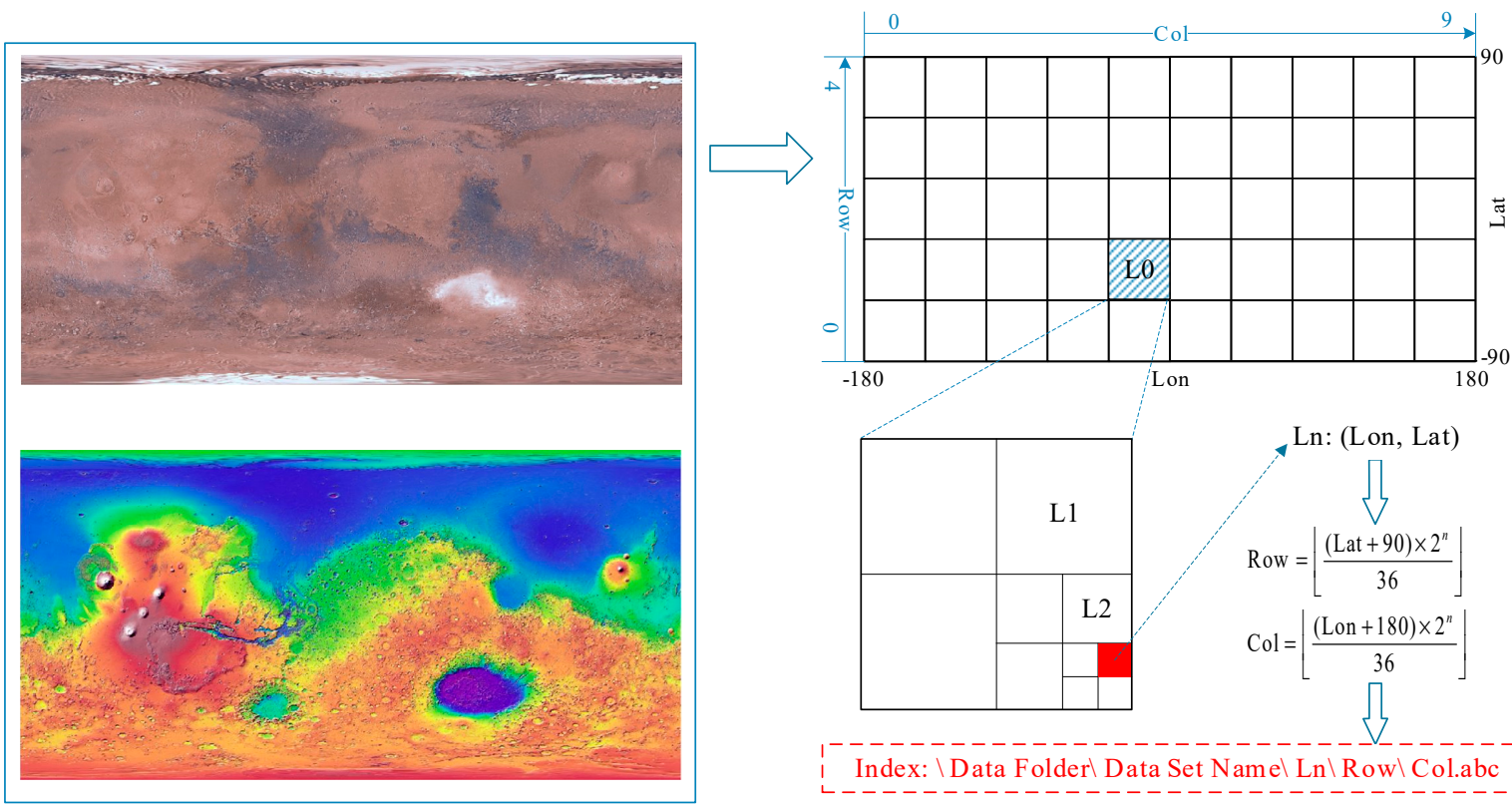

Index: \Data Folder $\backslash$ Data Set Name $\backslash$ Ln $\backslash$ Row $\backslash$ Col.abc

Figure 3. Geographic data segmentation and index method.

For example, considering the Mars images and DEMs, the size of the pyramid tile was set to $512 \times 512$ pixels, the first layer was divided into $5 \times 10$, and the row increased from $90^{\circ} \mathrm{S}$ to $90^{\circ} \mathrm{N}$ and the column increased from $180^{\circ} \mathrm{W}$ to $180^{\circ} \mathrm{E}$. Then, each tile was segmented layer-by-layer, and the row and column number (Row, Col) at the coordinate (Lon, Lat) of the Ln level could be calculated using the formula specified in Figure 3. The tile used three-level storage with the naming convention, 'Ln $\backslash$ Row $\backslash \mathrm{Col}^{\prime}$ ', which was convenient for querying and loading. For feature data, the point region (PR) quadtree index [60] was created based on the location coordinates and recorded using the eXtensible Markup Language (XML) file.

\subsubsection{Discrete Space Environment Data Index}

The space environment contains rich elements and covers a vast area; for example, taking the earth space environment as an example, elements such as the middle and upper atmosphere, the geomagnetic field, and the ionosphere are in the range of tens of kilometers to tens of thousands of kilometers. For space environment elements with a wide distribution and large density under a static state, whether it is the clipping in the visual process or the nearest neighbor in the query operation, the conventional traversal method does not meet the requirements. The spheroid degenerated-octree grid (SDOG) uses a volumetric discretization of the sphere that can provide a reference [61,62]. The octree spatial subdivision method was adopted to construct the index for the original version of Sino-InSpace (see Figure 4). 


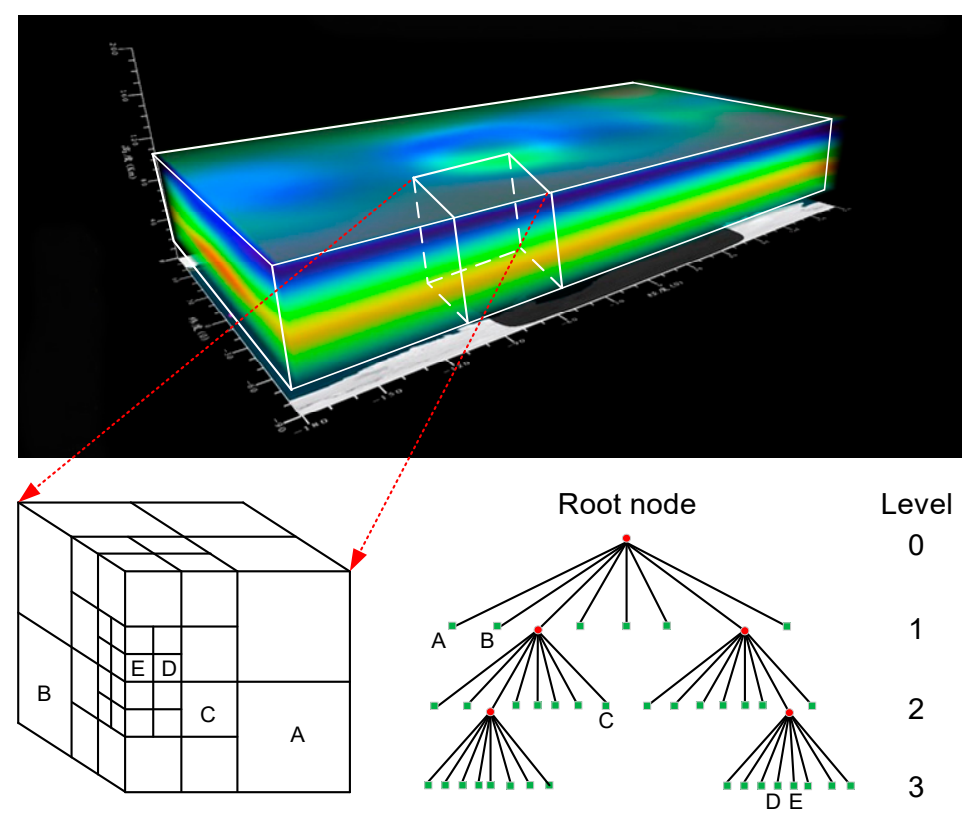

Figure 4. Space environment octree subdivision method.

An octree is a tree data structure involving spatial level recursive subdivision. The subdivision of the space environment data was based on the simple cylindrical projection plane and height direction. The cube represented by the root node included all the data to be processed. The root node was subdivided into eight sub nodes recursively, and the nonempty node continued to be divided. The maximum recursive depth of an octree depended on the resolution of various environmental data, display requirements, and query accuracy.

\subsection{Entity Object Model Design}

Satellites, rockets, and probes are used to carry out space missions such as launch, observation, and detection, and become the actual participants in space activities. Furthermore, these man-made space targets will become the main body of VSEs that needs to be supported and expressed because of the inability of a large number of human beings to enter into space at present. Figure 5 shows the four kinds of information required for describing a working BeiDou navigation satellite: (i) Attribute; this contains the name, id, category, and country of the object. (ii) Structure; this mainly describes the geometric form and the change. In addition to the model construction of the target, it also includes the change of overall form following the action of the components, such as the directional rotation of the solar panels during the operation. (iii) Position; determining the location of the target in the space-time coordinate system. The motion of the space target often follows a certain dynamic model. (iv) Attitude; according to the missions' need, the attitude of the satellite should be adjusted frequently such as the direction of the satellite antenna to the earth needs to ensure the visibility of the communication link. Therefore, a unified object model was designed, which consisted of the attribute, ephemeris, geometric model, and behavior model, which is shown in Figure 6.

Based on the multiple ephemeris segment, the ephemeris elements are used to determine the location of the target object at any time. Each ephemeris section contains a description of the timeline, coordinate system, and orbital information. In addition, three forms of orbits are supported in Sino-InSpace: Kepler, two-line element (TLE), and discrete track points. The first two orbits have their own prediction model which is used to calculate the position. For the description of the shape, the geometric model is composed of components that are constituted by some primitives based on the tree structure; the primitives are the smallest parts of the model consisting of dots, lines, planes, and textures. In addition, the primitive can also be a model file constructed by commercial software (e.g., 3ds Max). The behavior model is used to describe the movements of the overall and internal 
components of the target object at different stages. Each phase contains a description of the timeline, action object, and behavior type, which includes three types of operations such as translation, rotation, and scaling.

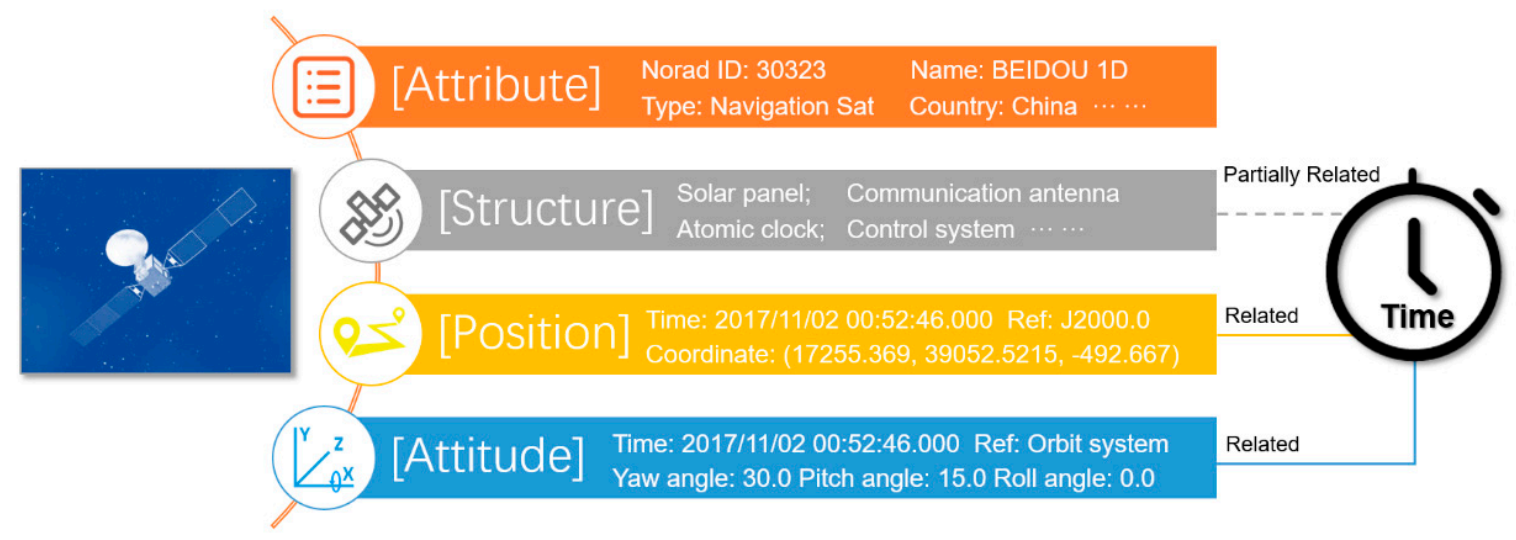

Figure 5. Description for a BeiDou navigation satellite. Attribute information does not generally change. Structure information is partially related to time because sometimes the components need to adjust. Position and attitude information is always related to time.

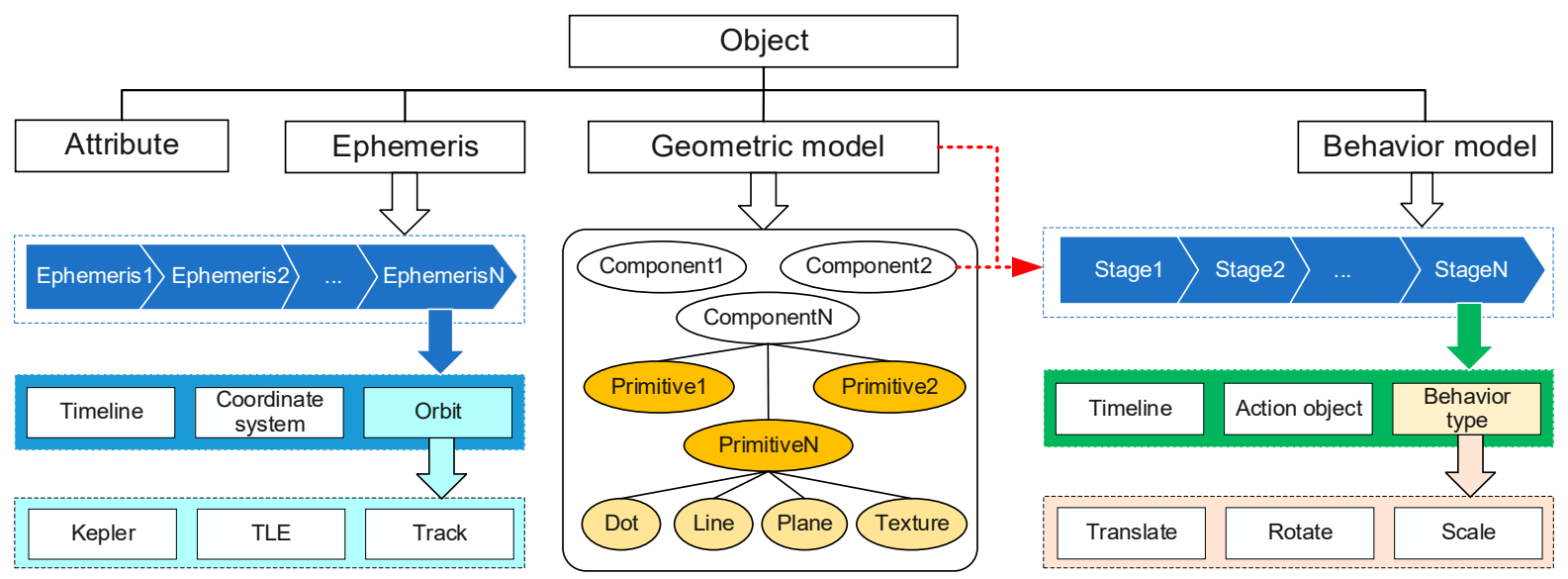

Figure 6. Entity object model design. The action object derives from the component or geometric model itself.

\subsection{Visualization Scenario Organization}

The visualization scenario refers to the organization of the geographical environment, entity and space environment elements, and space activities, which belong to the geographical scene. Traditional cartography and GIS are commonly used to express the static information and temporal relationship. The visualization scenario focuses on multilevel and multidimensional information regarding time, location, participants, events, processes, and phenomena, among others [38,63]. The visualization scenario organization in this study was based on two levels: logical modeling and data modeling, as shown in Figure 7. 
(a)

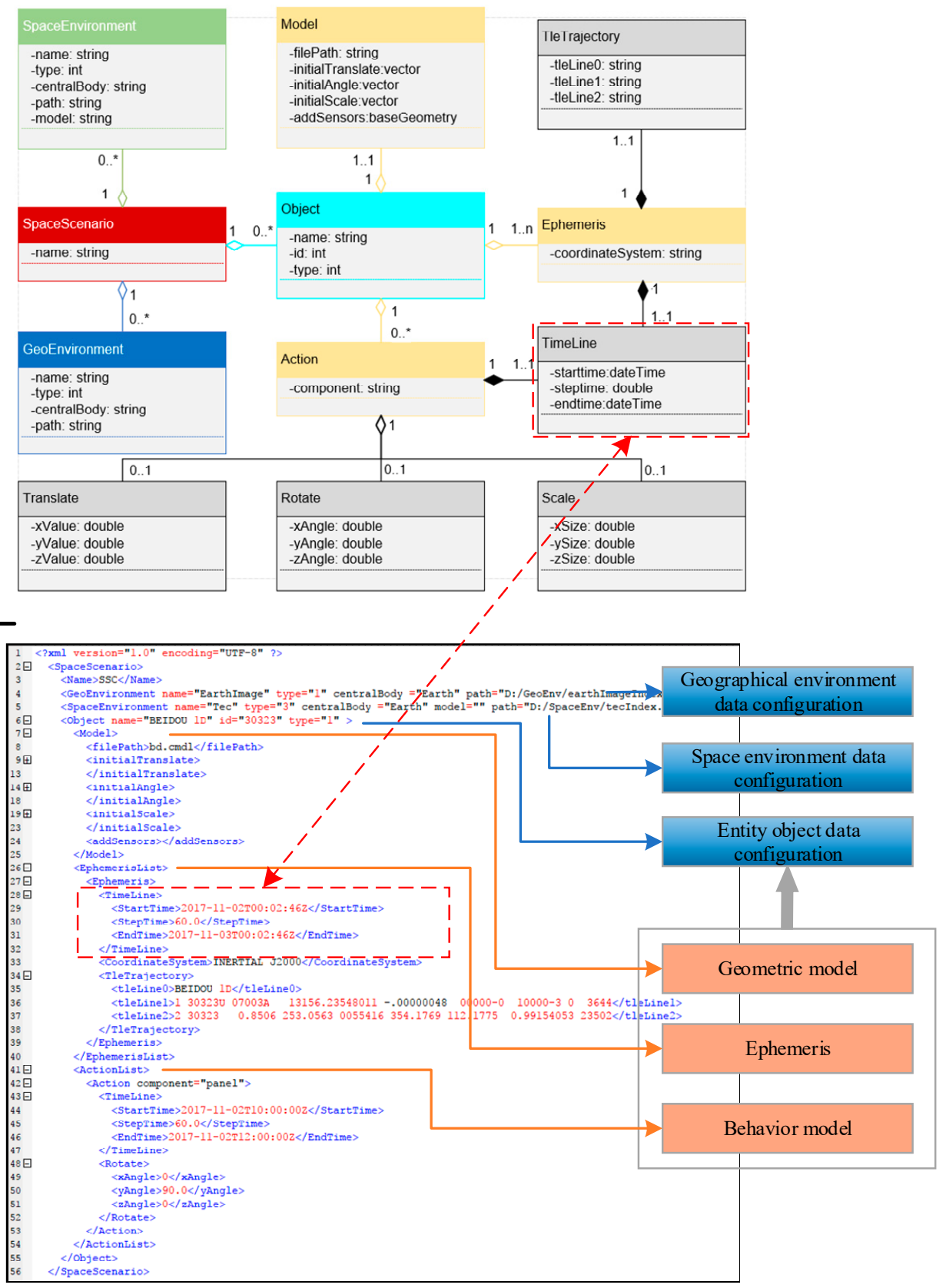

Figure 7. Scenario organization and modeling based on Unified Modeling Language (UML) and eXtensible Markup Language (XML). (a) UML diagram of the visualization scenario. (b) XML data of the visualization scenario. Timeline is used as an example; its logical model includes the start time, end time, and time step. The datatype of the start time and the end time is defined as dateTime, while that of the time step is Double. XML content was filled according to the field data type, which can be stored in the local file to be accessed later. Furthermore, Timeline was also the part of the Ephemeris and Action classes with the corresponding relationship of 1 to 1.

The static structure and relationship of the visualization scenario data were described by defining the specific terms using the Unified Modeling Language (UML) diagram. As in the previous case, SpaceScenario contains three main nodes: GeoEnvironment, SpaceEnvironment, and Object. The type, structure, data constraints, and the correspondence and association between different levels of the hierarchy were described. In GeoEnvironment, the path gives the file system information for the config file of the geographical data, which contains the details of pyramid tiles (e.g., region, tile size, 
tile level, and storage location). In SpaceEnvironment, the path and model indicate the source of the space environment data. The former refers to the directory of the environment data file and the latter is the model used for calculation. The Object node has three child nodes: Model, EphemerisList, and ActionList. These nodes depict the geometric model, ephemeris, and behavior model in Section 3.3. In Model, filepath means the path of the object geometric 3D model file. InitialTranslate, InitialAngle, and InitialScale describe the initial state of the object geometric 3D model. In EphemerisList, several Ephemeris nodes may be contained and each one represents a track. Here, the orbit type of "BEIDOU $1 \mathrm{D}^{\prime \prime}$ satellite is TLE. ActionList also comprises more than one Action node, which records the change of translation, rotation, and size.

\subsection{Visualization Engine Design}

The use of abstract data makes it difficult to meet the final needs of the users. However, the visualization technology can describe the data graphically to provide users with a visual outlook of these data, which can help them understand and recognize the current space activities. Faced with complex, massive spatial data, efficient data scheduling and flexible operational control are key problems in the visualization process. Therefore, Sino-InSpace divides the visualization engine into two parts: the data engine and rendering engine, as shown in Figure 8.
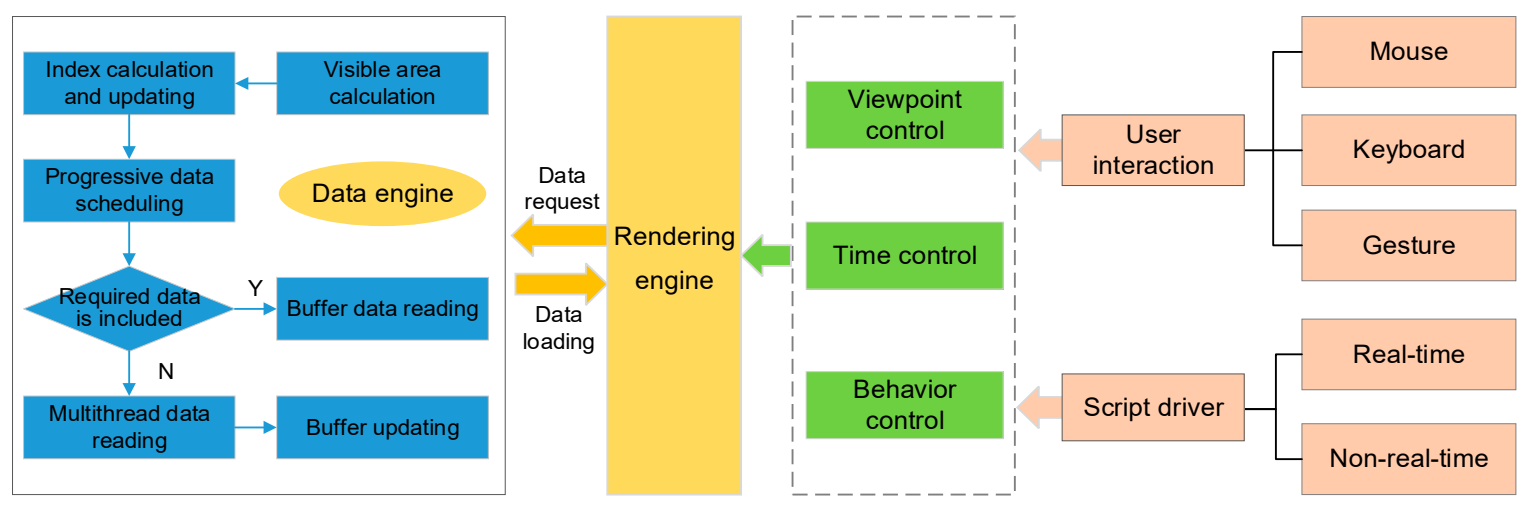

Figure 8. Visualization engine design.

The data engine is responsible for the rapid indexing and scheduling of data. Depending on the visual cone parameters delivered by the rendering engine, the visible area is first calculated and then, the tiles, environment data, or graphic names that need to be updated are determined. To improve the rendering of visualization, the data are progressively scheduled from near to far based on the distance to the viewpoints; in addition, a certain part of the data buffer memory is reserved for visualization. For the visualization process, first, we determined whether the data buffer contained the required data; if the data were not available in the buffer, they were imported by multithreading, and the data buffer was then updated. After receiving the data transmitted by the data engine, the rendering engine graphically renders the environment and entity elements in the visual scene.

The rendering engine also supports user interactions such as mouse-, keyboard-, and gesturebased interactions as well as real-time or non-real-time script drivers to flexibly control the viewpoint, time, and entity target behavior. A script file can be opened and edited directly in text, and each line represents a command and has a fixed format as follows:

\section{Command identification $\{[$ Parameter identification Parameter $]\}$}

Table 2 shows some of the commands and parameters. These commands support the switch of viewpoints, control of simulation speed, text display, etc. A simple example is given in Figure 9. 
Table 2. Some commands and parameters.

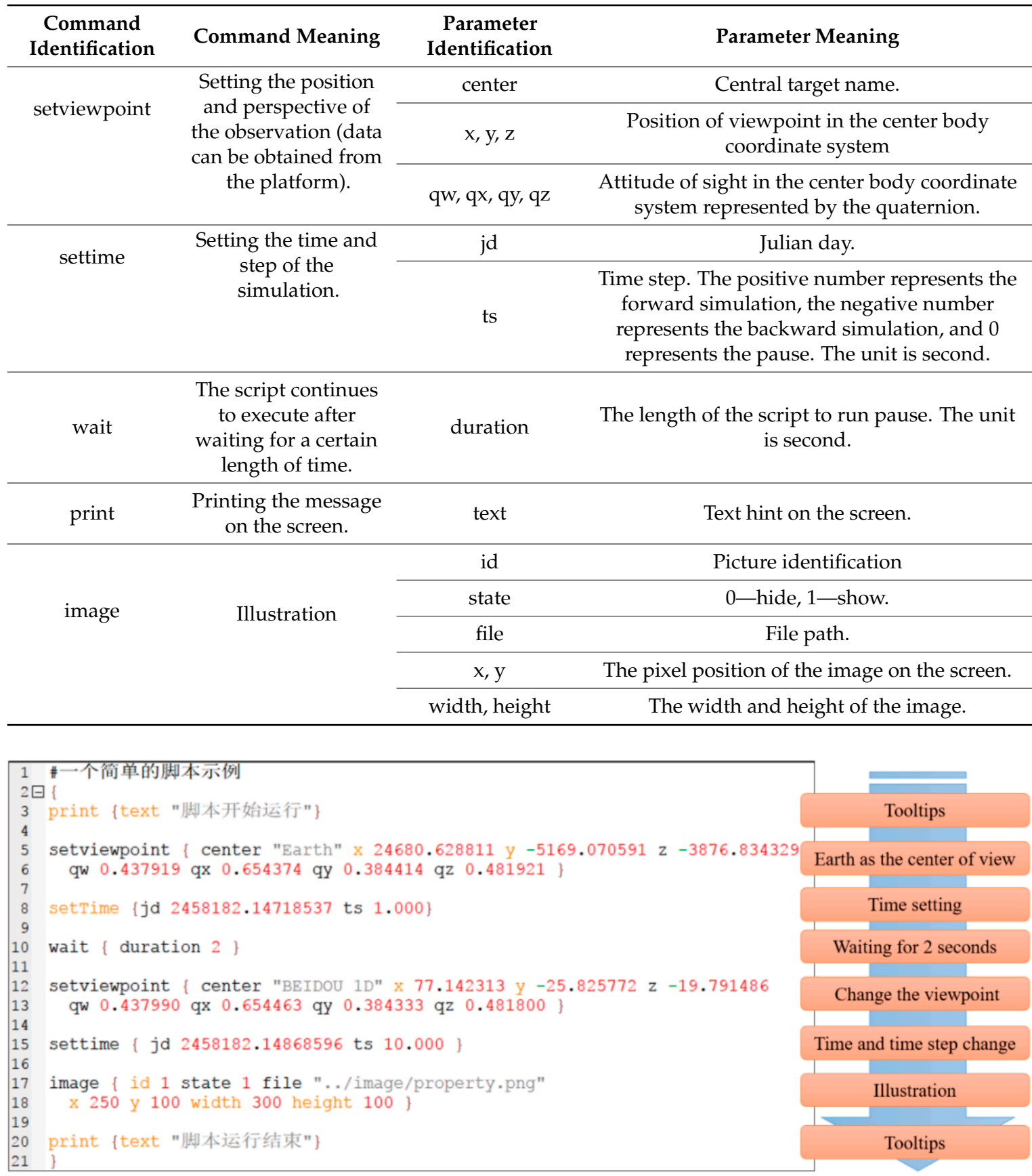

Figure 9. A simple example of the script file.

\section{Design of Platform Application Modes and Case Study}

By supporting geo-visualization, geo-simulation, geo-collaboration, and human participation, VGEs provide open virtual environments that correspond to the real world to assist computer-aided geographic experiments [8]. As a digital simulation platform for VSEs, Sino-InSpace aims to satisfy the multilevel requirements of the application. As shown in Figure 10, three modes are supplied including application directly (without more development), visualization development, and scientific analysis. 
- Data integration and visualization

- Space mission simulation and deduction

- Popular science

\section{Application Directly}
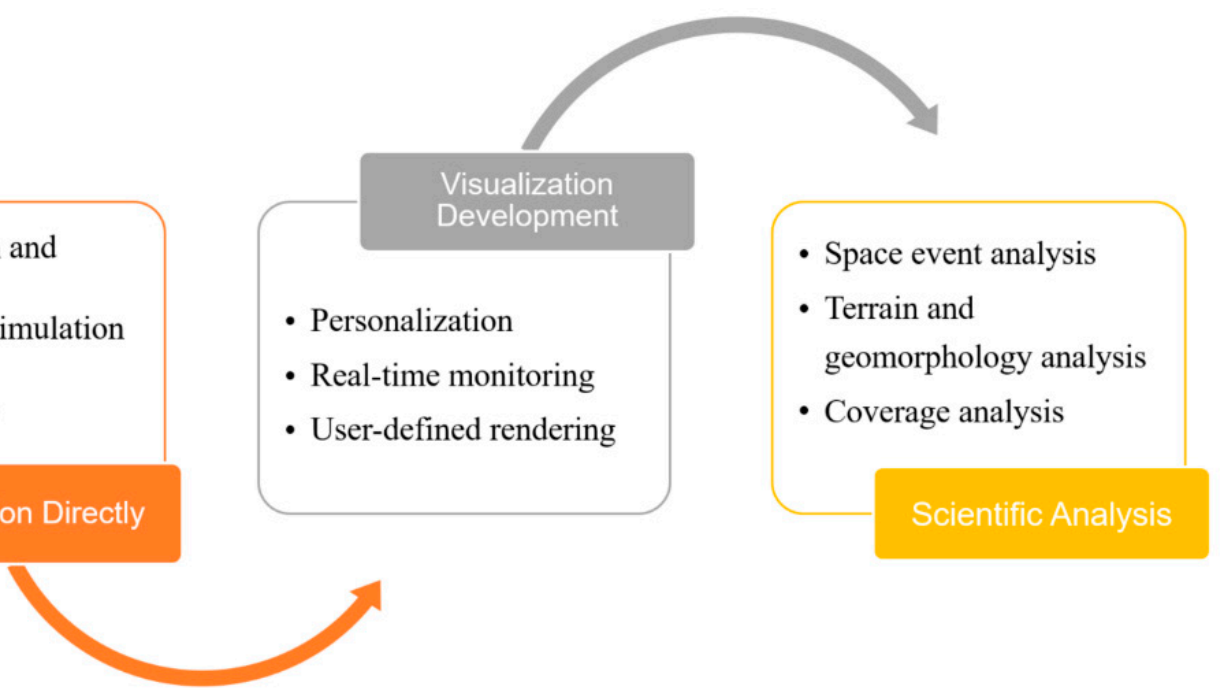

Figure 10. Design of platform application modes.

\subsection{Application Directly}

Sino-InSpace provides an executable software to serve low-level users directly. It has an accurate starry sky and can integrate massive environment data from different planets. Therefore, it is possible for children to study the solar system and for the public to understand the appearance of other planets. In addition, complex simulation and deduction of space missions can be conducted through the scenario construction and script edit. Finally, the virtual reality setup combined with Sino-InSpace in our laboratory was introduced and used to evaluate the performance of the platform. All case studies in this section were conducted with a Dell workstation (Intel Core i7 CPU, 8G RAM, Nvidia Quadro K2000 graphics card, and Microsoft Windows $10 \times 64$ operating system).

\subsubsection{Application Main Window}

The application main window is shown in Figure 11 and offers a friendly operation interface including the Scenario Editor, 2D and 3D view, Time Control, and Viewpoint Control. Scenario Editor provides the structure of scenarios constructed with satellites, spacecraft, environment configurations, and so on. Entity elements including properties, ephemerides, geometric models, and actions (mentioned in Section 3.3) can be edited further. Multidimensional visualization enables the presentation of scenarios and phenomena to be more dynamic [64]. The platform provides 2D and $3 \mathrm{D}$ view windows. The mouse and keyboard can be used for smooth panning and zooming through data with six degrees of freedom. The Viewpoint Tool helps to add, edit, delete, and switch between different viewpoints; this is convenient for flexible observations. Furthermore, the Time Tool contains the function of acceleration, deceleration, going ahead, going back, and time setting to satisfy browsing the event at different speeds and times. 


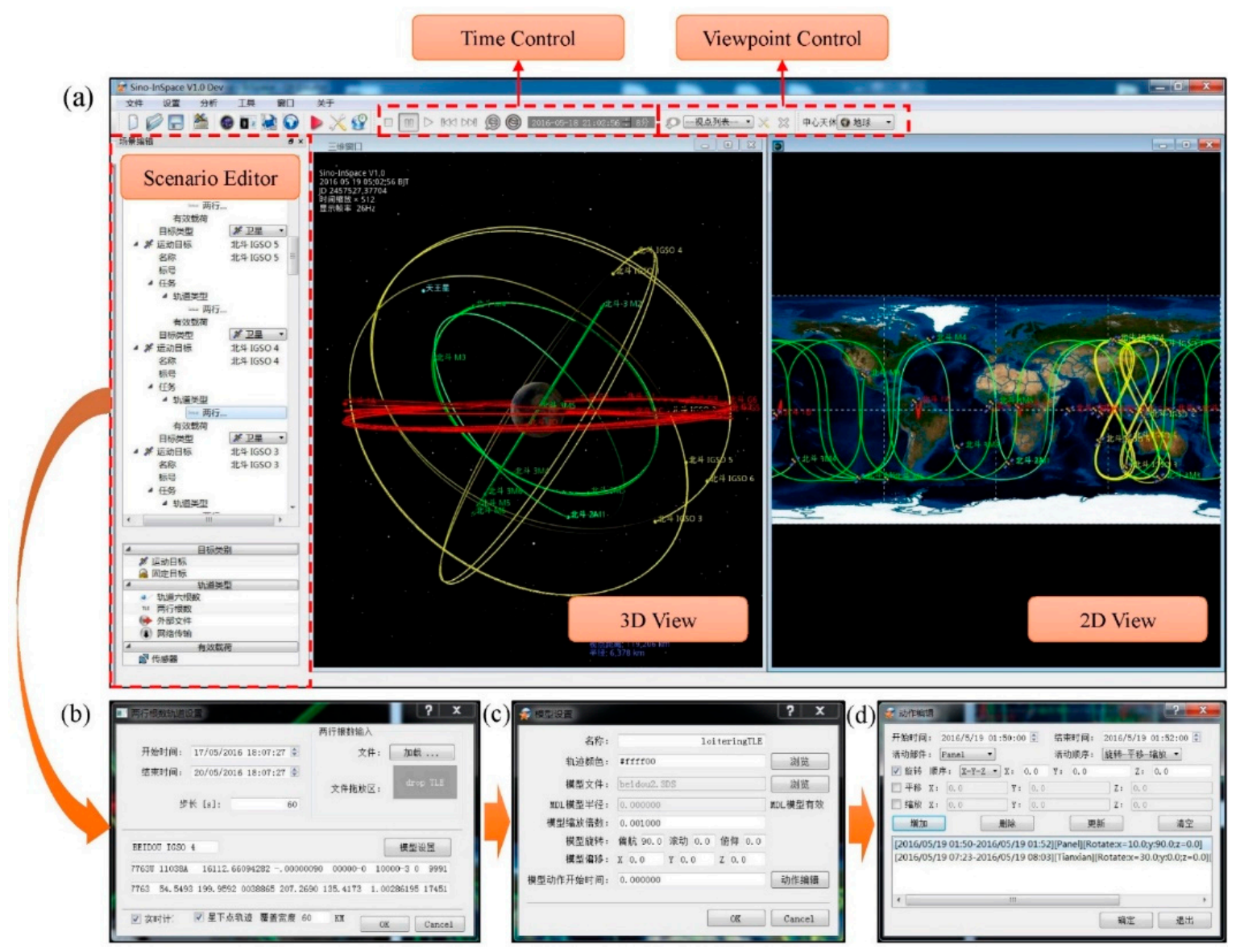

Figure 11. The operation window of Sino-InSpace in the application directly mode. (a) The main window includes the Scenario Editor, 2D and 3D view, Time Control, and Viewpoint Control; (b) Trajectory setting window; (c) Geometric model setting window; and (d) Action design window.

\subsubsection{Visualization and Deduction}

Sino-InSpace provides a flexible and visible platform for the massive achievement not only on earth but also on other planets. In support of a perfect time-space datum framework, stars, planets in the solar system, and geographical and space environment data are integrated, organized, and displayed as shown in Figure 12. Excellent data organization and a good visualization engine guarantee the fluent running of the platform. The time delay mainly originates from the initial data loading and the real-time calculation (e.g., orbit prediction and empirical space environment models). The time required to load the necessary data in the illustrations is no more than $3 \mathrm{~s}$, and the frame rates are all greater than $30 \mathrm{ftps}$, which is sufficiently satisfactory for observation.

Advance deductions are often carried out to enhance the understanding of the mission. As shown in Figure 13, a launching mission deduction of the Shenzhou spacecraft was designed. The trajectory and action data of the $\mathrm{CZ}$ rocket and manned spacecraft were added to the related object model. Then, the script file was edited and loaded to drive the mission execution, and the viewpoint was switched to the interested perspective. Meanwhile, some illustration and text hints appeared on the screen occasionally. 

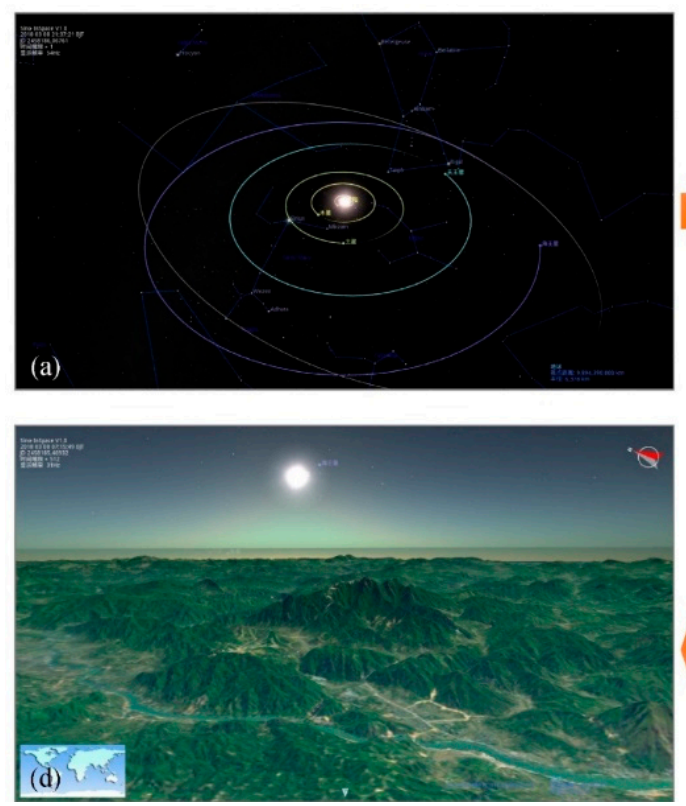

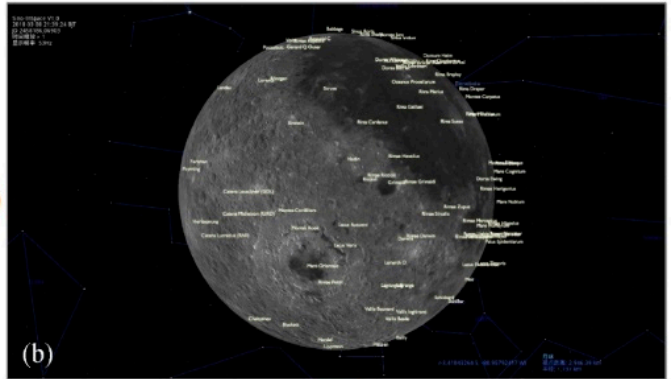

ए

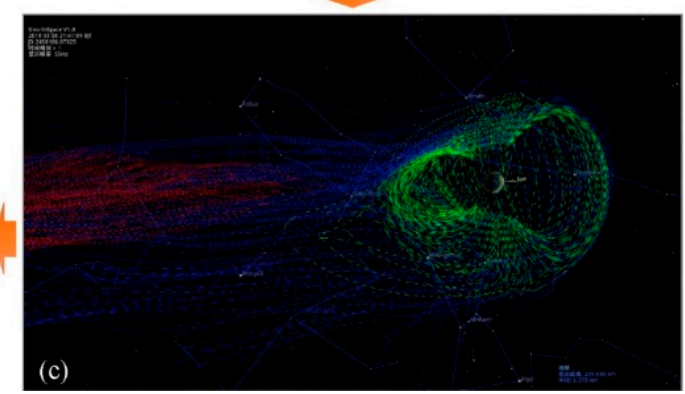

Figure 12. Integration and visualization of the constellation and environment data. (a) Stars (Tycho-2 catalogue, number: $>2.5$ million) and planets in the solar system (NASA Jet Propulsion Laboratory Development Ephemeris (JPL DE) 405); (b) all named features for the moon (number: 8990) [65]; (c) geomagnetic field generated from the Tsyganenko 96 model [66], which includes the solar-wind-controlled magnetopause, region 1 and 2 Birkeland currents, and the interconnection of the magnetospheric and solar wind fields at the boundary; and (d) geographical data from the southeast area of China (image resolution: 30 m, DEM resolution: 90 m, data amount: >200 GB).

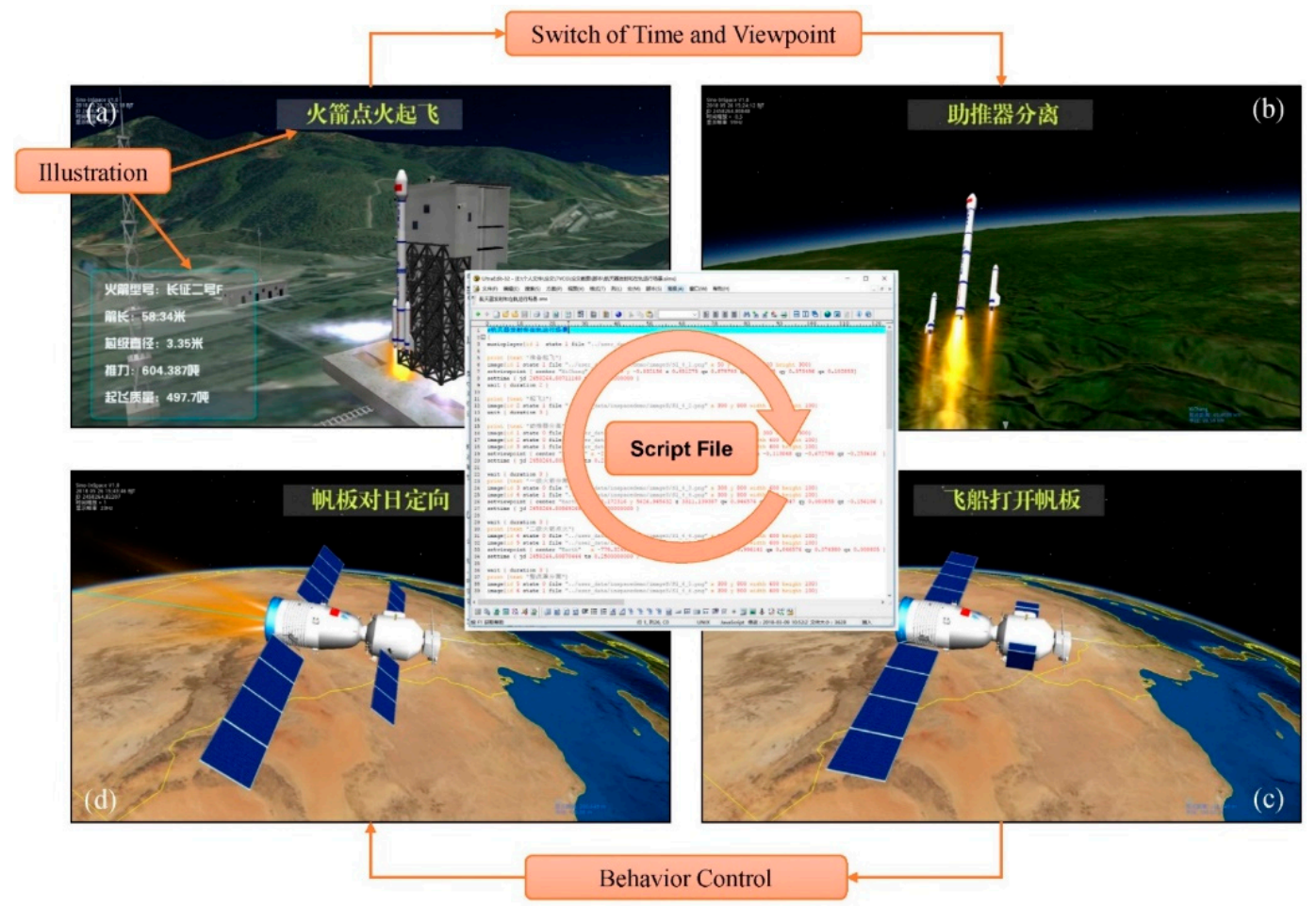

Figure 13. Launching mission deduction of the Shenzhou spacecraft. (a) Rocket firing. The illustrations introduced the stage name and the capacity of the $\mathrm{CZ}$ rocket. (b) Rocket booster separation. The separation action executed $114 \mathrm{~s}$ later; (c,d) the process of solar panel expansion and orientation. The solar panel as a component of the spacecraft model was translated and rotated according to the action sequence setting. 


\subsubsection{Virtual Reality Setup}

Virtual and augmented reality have received a considerable amount of attention by researchers in the last decade and provide new ways to perceive the world [67-69]. Virtual reality is defined as a computer-generated scenario that simulates experience through senses and perception [70], while augmented reality creates an environment where digital information is inserted in a predominantly real-world view [71]. The well-known basic components of a VR immersive application include input devices (responsible for interaction), output devices (for the feeling of immersion), and software (for a proper control and synchronization of the whole environment). For the better simulation and presence for VSEs, a simple VR setup was constructed in our Space Situational Awareness (SSA) shared laboratory based on Sino-InSpace. The standard mouse and keyboard allow users a relatively easy and normal way of manipulation of a virtual scenario. In addition, Sino-InSpace supports a variety of stereoscopic observations (e.g., red and green, split screen, and blinking) to produce an immersive environment. The first two types have no special requirement for the hardware, and the virtual experience is poor accordingly. The blinking mode is most frequently used with the help of Nvidia 3D vision technology (Figure 14). The output devices consist of a stereoscopic graphic card (Quadro K2000), synchronous signal transmitters, 3D glasses, Panasonic projectors, and a projection screen. The equipment supports up to tens of people viewing stereo content simultaneously, which could better assist in collaborative and multiperspective space science research and education.

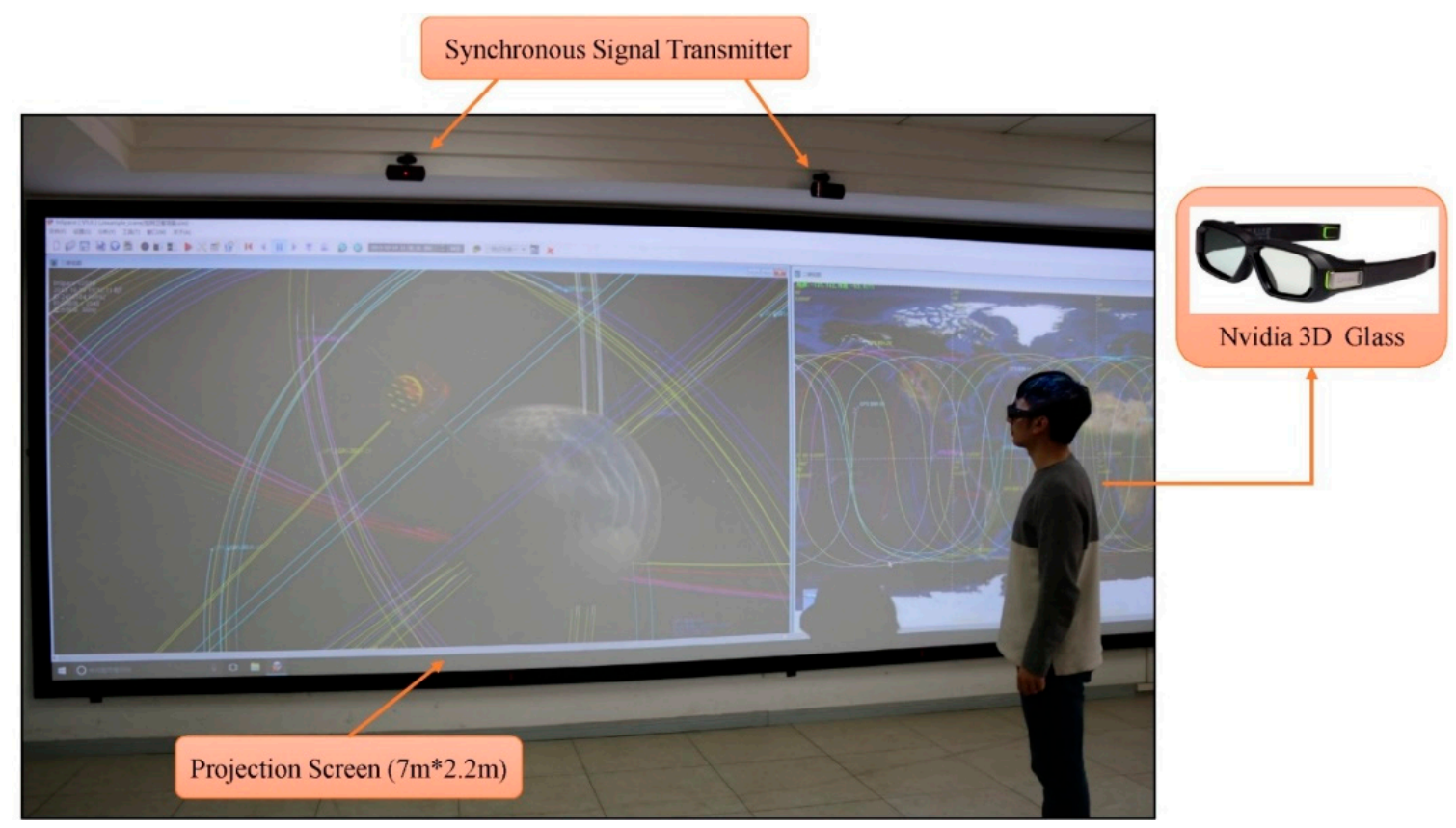

Figure 14. Blinking stereoscopic observations with the help of Nvidia 3D vision technology. The synchronous signal transmitters control the switch of two lenses of 3D glasses, while the 3D view window provides a left or right frame.

\subsection{Visualization Development}

Compared with the "Application Directly" mode, the "Visualization Development" mode was proposed to develop the individualized customizable software. Figure 15 presents the core components of Sino-InSpace. The SSimAstroCore component is mainly used to provide mathematical functions and model calculations, e.g., coordinate transformation, matrix operation, orbit prediction, space environment model calculations, and so on, which are the basis of the platform. The SSim2DView and SSim3DView components, as the visualization engines, are responsible for the data loading and rendering. The SSimScenario component parses the scenario file and extracts different types of elements for the next step. The SSimMainApp component is the interface between the external program 
and internal components and is the most frequently used component in "Visualization Development" mode. It supplies encapsulated User Interface (UI) widgets shown in Figure 11 which can be embedded in the new program conveniently. In addition to the related functions such as environment data and objects adding and time and viewpoint control, local scenario file open was also developed.

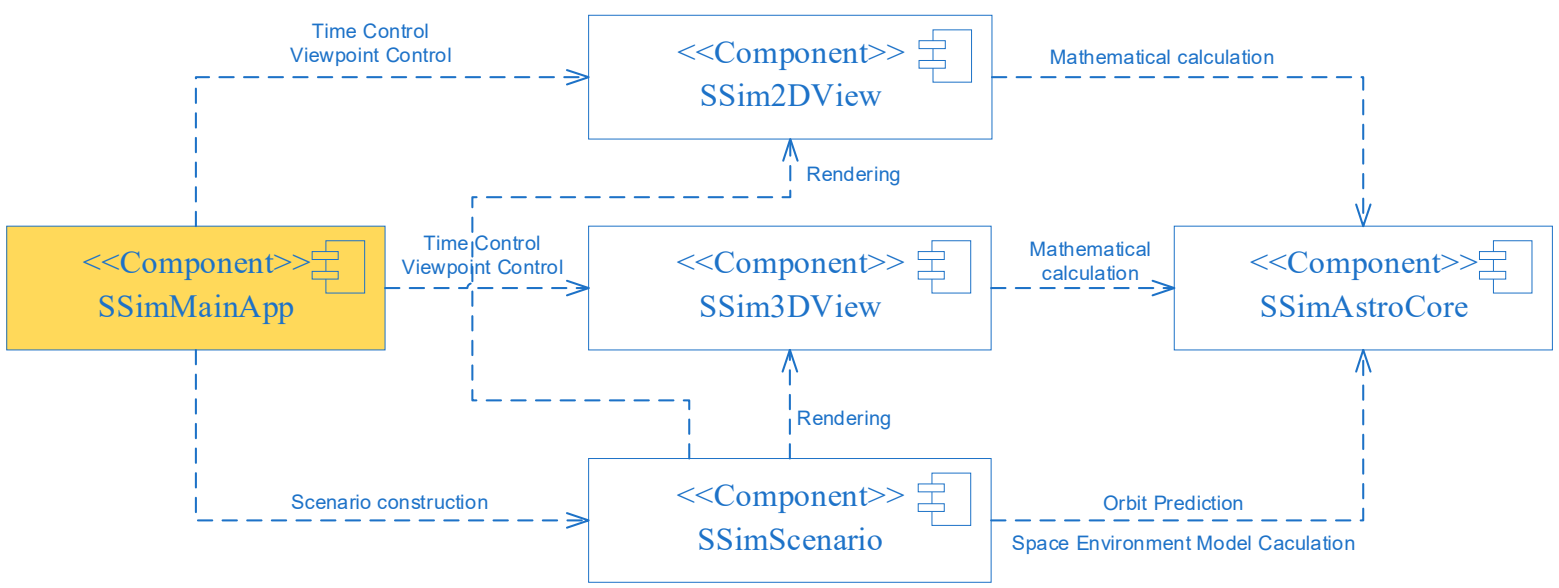

Figure 15. Core component diagram of Sino-InSpace. Arrows indicate dependencies.

Taking the "Comprehensive Situation System" in the China BeiDou Navigation Satellite System (BDS) data center as an example (Figure 16), it was developed based on Sino-InSpace in the "Visualization Development" mode. The offline and online location data, location device situation, BeiDou satellite situation, and map service were passed into the platform. The SSimMainApp component provides the interface for the access to these entity objects and updates their trajectory and state changes in real time. For the map service following the Web Map Service (WMS) standard, the component will obtain the image tiles under the control of Data Engine (Section 3.5). In addition, the software style was customized. Some UI widgets (time and viewpoint control tools) were invoked directly. As the platform was developed under Qt languages, a readable and declarative language, the Qt Meta-Object Language (QML) [72] is often adopted to create fluidly animated and visually appealing applications. It allows components to be easily reused and customized within a user interface.

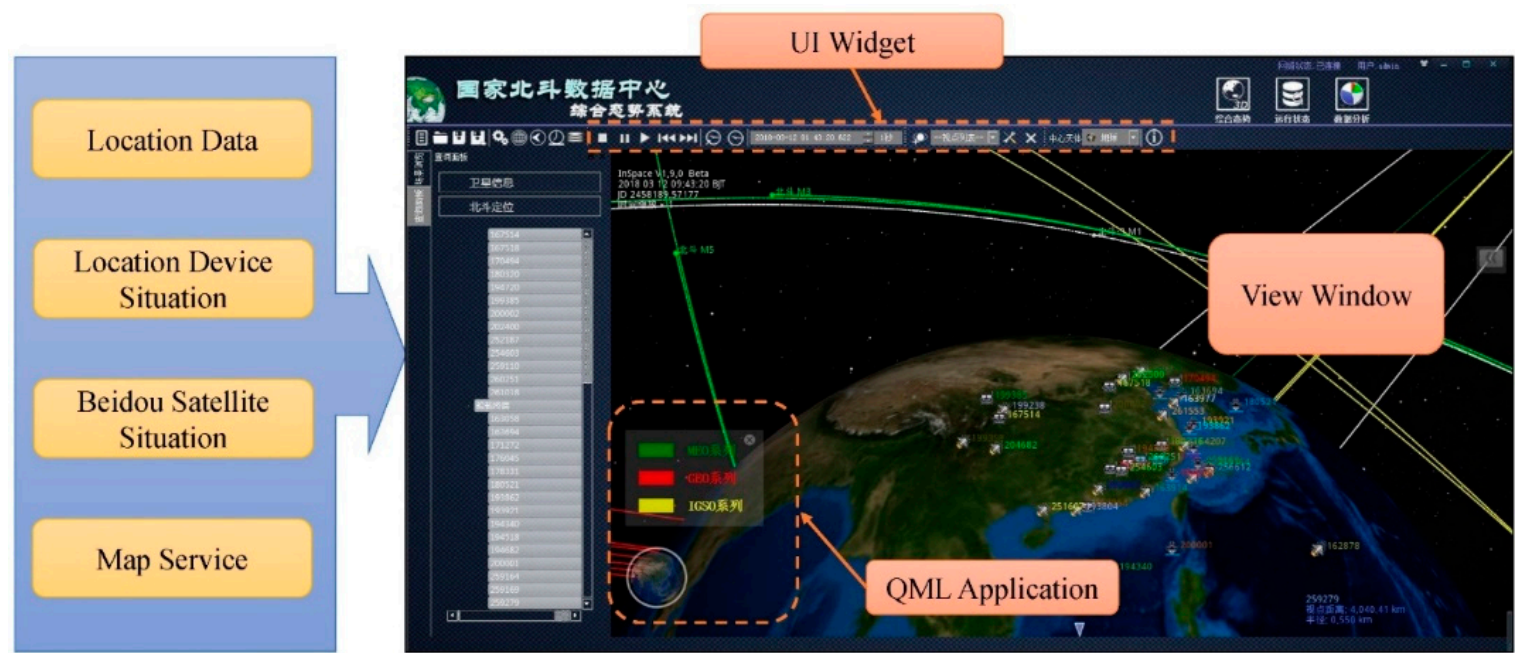

Figure 16. Comprehensive situation system based on Sino-InSpace. Qt Meta-Object Language (QML) was used for the interactive operation and information display.

The entity object usually has a specific and given geometry that can be created or combined in advance. However, some invisible or changing parameters such as sensor range, communication 
link, and space environment boundary also need a visible and dynamically generated geometry. It is impossible for any visualization platform to integrate all shapes, including Sino-InSpace. Therefore, it provides the "BaseGeometry" class in the SSim3DView component, which can be inherited for rendering. Any geometry drawn with a standard OpenGL language can be added to the geometric model (Figure 6) of any entity object. This function maximizes the extensibility of the platform. Figure 17 illustrates a visualization result for the Van Allen radiation belt in cooperation with the National Space Science Center (NSSC) and Chinese Academy of Sciences (CAS). The distribution and flux of the radiation belt particles were calculated by the AE8/AP8 model [73], which was expressed with boundary lines and colors, respectively.

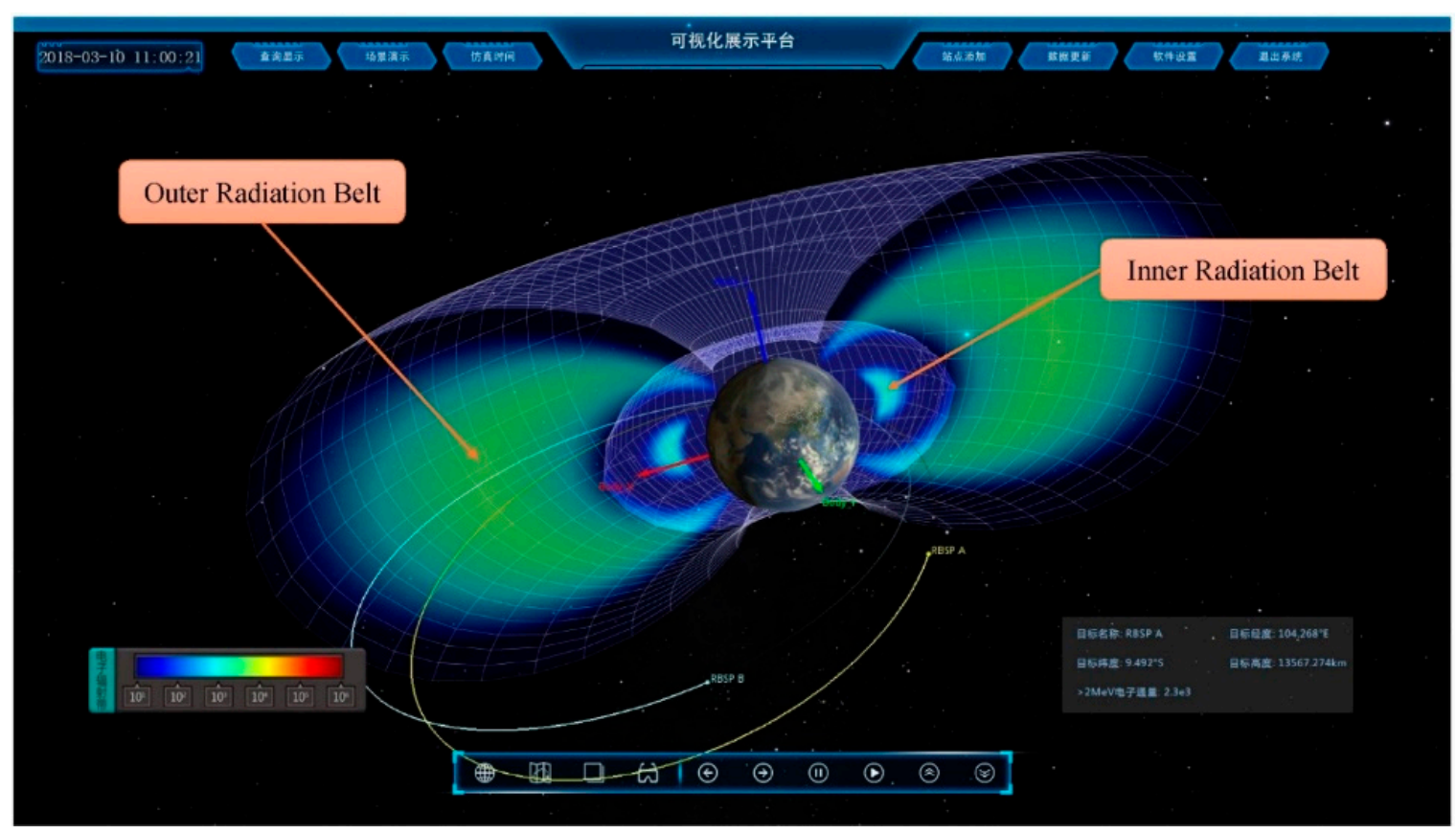

Figure 17. Van Allen radiation belt provided by the AE8/AP8 model.

\subsection{Scientific Analysis}

As a platform for data import and visualization, Sino-InSpace also opens interfaces to export calculation and query results. The SSimMainApp component (Figure 15) provides most of the output interfaces, mainly divided into three types: system information (time, viewpoint, mouse position, etc.), environment data (position, distribution, temperature, intensity, etc.), and entity object data (attribute, structure, position, and attitude). The latter two types are meaningful for scientific analysis. The analysis of space objects collision warning was carried out as shown in Figure 18. The orbit and position data were obtained to estimate the most likely approach and even collision events outside the platform [74]. In contrast, the result could be validated through the reappearance of collision scenario. The distances in three orthogonal directions (radial, in-track, and cross-track) between two risk targets were plotted as curves. The closest time was clear with the help of visual analysis.

To support the Chinese Mars Exploration Project 2020, China's Ministry of Science and Technology funded research on planetary surface precise landing guidance and control (Grant No. 2012CB720000-G) during the twelfth five-year plan. The landing area selection is one of the most important scientific objectives. Based on the strategy of the multiresolution pyramid model (Figure 3), the altitude could be obtained in real time. Then, terrain analysis tools (Figure 19) containing point, distance, area measurement, and profile-map were developed, which supported mouse selection and manual input. It played a role in the terrain and geomorphology analysis of the Mars surface. 

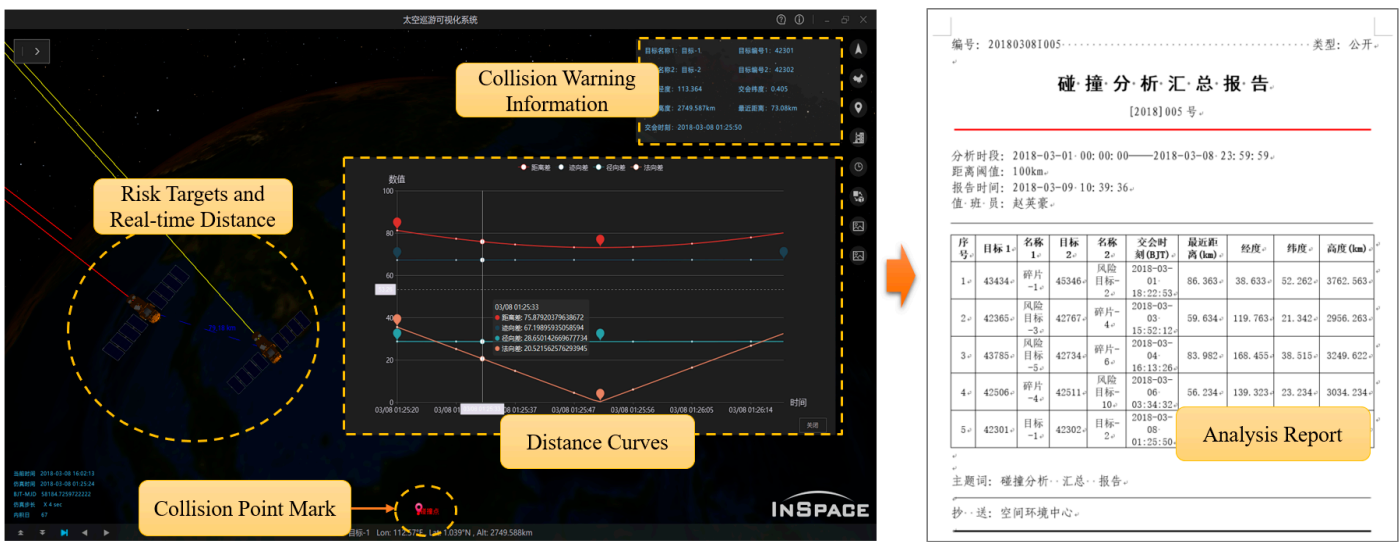

Figure 18. Space objects collision warning analysis and report output. The distance curves are drawn with ECharts [72], a powerful, interactive charting and visualization library for browsers.

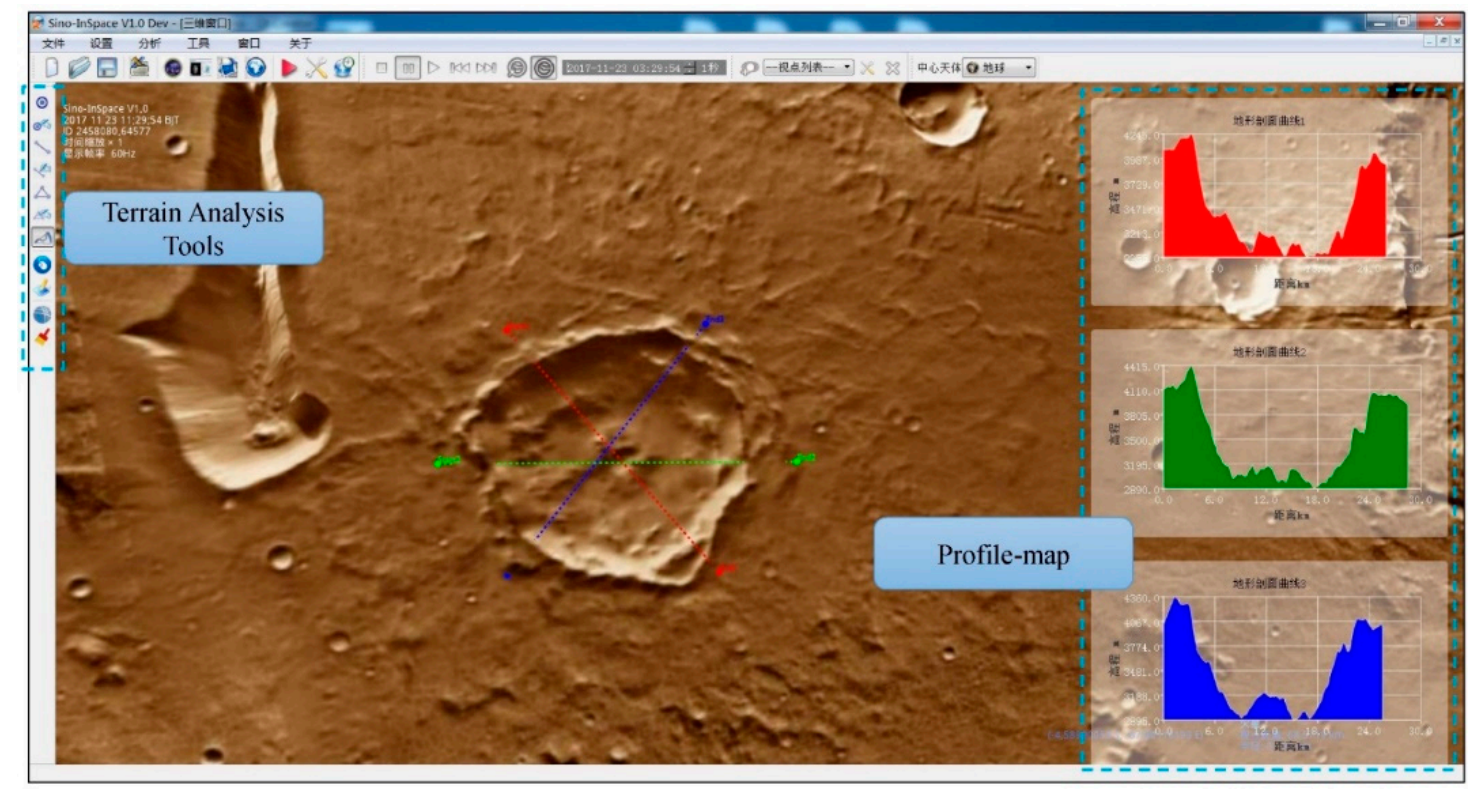

Figure 19. Terrain and geomorphology analysis. The crater closest to Ceti Chasma. The base map was derived from Mars Colorized Viking Mosaic [75] and the resolution is $232 \mathrm{~m}$. The source of DEM is MOLA MEGDRs [76] and the resolution is $463 \mathrm{~m}$. The amount of segmented geographical data exceeds 15 GB.

\section{Platform Evaluation}

Despite the fact that Sino-InSpace provides real-time interactive experiences via computer simulations, little is known about whether potential users perceive VSEs effectively and how user-system interaction contributes to system usability. Many Human-Computer Interaction (HCI) studies have compared learning effects, performance, and subjective ratings with different applications and tasks [77], which provide grounds for an evaluation of the system.

The different approaches of measurement fall into two general categories: subjective measures and objective measures. Here, a user study with human participants was conducted using postquestionnaires, which are the most common technique in subjective measurement. Usability, user acceptance, and presence are considered based on previous research $[78,79]$ to understand and evaluate critical contributions. Each questionnaire contains 30 items regarding usability (10 items), user acceptance (10 items), and presence (10 items) as listed in Appendix A. The experience in stereo 3D (blinking), nonstereo 3D (naked-eye), and 2D conditions were tested together using the virtual reality setup. In addition, the latter two application modes related to development were also studied 
from the perspective of software design [80]. Each questionnaire consists of 10 items considering correctness ( 2 items), robustness ( 2 items), flexibility ( 1 item), reusability ( 2 items), efficiency ( 1 item), reliability (1 item), and usability (1 item) as listed in Appendix B. All the answers for each question are marked on a five-point scale except for user acceptance (seven-point scale).

\subsection{Hypotheses}

The performance of Sino-InSpace was measured by two questionnaires from different perspectives. However, all questions are in the same and positive direction. The higher score indicates better performance. Naturally, we expect that performance in an upper level and multidimensional condition contributes to a better experience. Accordingly, the hypotheses are:

Usability, user acceptance, and presence in three conditions (i.e., stereo 3D, nonstereo 3D, and 2D) will be at a good level ( $>80 \%)$. In other words, usability and presence ratings will exceed 40 , while the user acceptance rating will exceed 56.

Usability, user acceptance, and presence ratings will decrease in the sequence of stereo 3D, nonstereo 3D, then 2D conditions.

The software design rating of the platform will exceed 40.

\subsection{Participants and Procedure}

In testing the first two hypotheses, the participants were composed of 27 undergraduate students, 12 graduate students, and six professors from a large university in China who had never used Sino-InSpace. In terms of gender, 10 were female and 35 were male. Their ages were between 19 and 54 years. The same procedures and instruments were used for stereo 3D (blinking), nonstereo 3D (naked-eye), and 2D conditions. The participant numbers were balanced among the three groups.

A brief instruction for the mouse and keyboard control, time, and viewpoint control, and scenario and script file edit were first provided. There were $10 \mathrm{~min}$ for each participant to become familiar with the environment and operations. Then, three tasks relating to key technologies of the platform were carried out.

Multiscale Environment Experience: Participants travelled between different planets in the solar system freely. They evaluated the efficiency of data loading, the effect of visualization, and the flexibility of the interactive process.

Scenario Construction: A simple scenario, such as the one Figure 7 described, was assumed. Participants were instructed to complete the whole process of construction (new-built, elements adding, entity object model edit, save, etc.) and evaluated the usability of the platform.

Deduction Script Design: Based on the above scenario, participants designed the deduction script (such as in Figure 9) as they expected to test the applicability of the visualization engine.

A paper questionnaire (Appendix A) was administered immediately after each experiment. Participants were asked to rate their response or agreement with the statements with regard to how it describes their experience. Finally, the results were collected in the computer for statistical analysis.

Considering the long learning cycle of platform development, 14 participants from our cooperative institutes and companies overall, who had developed related applications using Sino-InSpace, took part in the study for hypothesis 3. An email questionnaire (Appendix B) was sent to them and returned after being filled out based on the actual experience. Then, the ratings were gathered for statistical analysis.

\subsection{Evaluation Result}

All participants successfully completed designated tasks. Overall, 59 cases were collected for analysis, which was performed with SPSS 17.0. First, the internal consistency of the measurement scales was assessed using Cronbach's alpha [81]. All questions are in the positive direction and the scores of Cronbach's alpha are as follows, usability $=0.91$, user acceptance $=0.85$, presence $=0.93$, and software design $=0.91$. The scores for the four variables all exceeded 0.80 (the generally accepted level), which indicated that measures of the associated constructs in the study were internally consistent. 
To determine the distribution of variables, normality tests were first conducted. Table 3 shows the Shapiro-Wilk (SW) test statistics and probabilities of the different measures in the three conditions. Software design was also tested $(W=0.973, p=0.904)$. However, the data across different conditions did fit well with a normal distribution $(p>0.05)$, which provided a premise and foundation for the mean test analysis.

Table 3. Normality test results on stability, user acceptance, and presence.

\begin{tabular}{ccccccc}
\hline \multirow{2}{*}{ Measure } & \multicolumn{2}{c}{ Stereo 3D } & \multicolumn{2}{c}{ Nonstereo 3D } & \multicolumn{2}{c}{ 2D } \\
\cline { 2 - 7 } & $W$ & $p$ & $W$ & $p$ & $W$ & $p$ \\
\hline Usability & 0.983 & 0.984 & 0.958 & 0.657 & 0.957 & 0.648 \\
User & 0.989 & 0.999 & 0.984 & 0.989 & 0.984 & 0.991 \\
acceptance & 0.969 & 0.837 & 0.969 & 0.836 & 0.984 & 0.973 \\
Presence & 0.969
\end{tabular}

Hypothesis 1 and hypothesis 3 predicted that ratings would be at a good level in each condition. Considering the number of cases $(<30)$ and the unknown variance, lower-tail one-sample t-tests were used to compare the means with the expected values. Participants perceived a great level $(p>0.05)$ of usability and presence in the stereo 3D and nonstereo 3D conditions, as shown in Table 4 and Figure 20. However, ratings of user acceptance in three conditions and measures in the 2D condition were significantly lower $(p<0.05)$ than the expected values. The results indicated that more effort should be devoted to creating user-friendly application interactions and interfaces. Meanwhile, there is no high-level perception of VSEs in the 2D condition. For software design, participants gave a high rating $(M=40.27, S D=1.94, T=53, p=0.70)$, which proves the ability of Sino-InSpace as an extensive future application.

Table 4. Usability, user acceptance, and presence mean comparisons using a lower-tail one-sample $t$-test.

\begin{tabular}{ccccccccccccc}
\hline \multirow{2}{*}{ Measure } & \multicolumn{4}{c}{ Stereo 3D } & \multicolumn{4}{c}{ Nonstereo 3D } & \multicolumn{4}{c}{ 2D } \\
\cline { 2 - 12 } & $\boldsymbol{M}$ & $\boldsymbol{S D}$ & $\boldsymbol{T}$ & $\boldsymbol{p}$ & $\boldsymbol{M}$ & $\boldsymbol{S D}$ & $\boldsymbol{T}$ & $\boldsymbol{p}$ & $\boldsymbol{M}$ & $\boldsymbol{S D}$ & $\boldsymbol{T}$ & $\boldsymbol{p}$ \\
\hline Usability & 41.13 & 2.90 & 1.51 & 0.92 & 40.27 & 3.54 & 0.29 & 0.61 & 32.20 & 3.75 & -8.07 & 0.00 \\
User acceptance & 45.67 & 5.08 & -7.88 & 0.00 & 45.00 & 5.18 & -8.22 & 0.00 & 44.47 & 4.66 & -9.59 & 0.00 \\
Presence & 42.80 & 3.40 & 3.18 & 1.00 & 39.53 & 3.16 & -0.57 & 0.29 & 33.60 & 3.09 & -8.02 & 0.00 \\
\hline
\end{tabular}

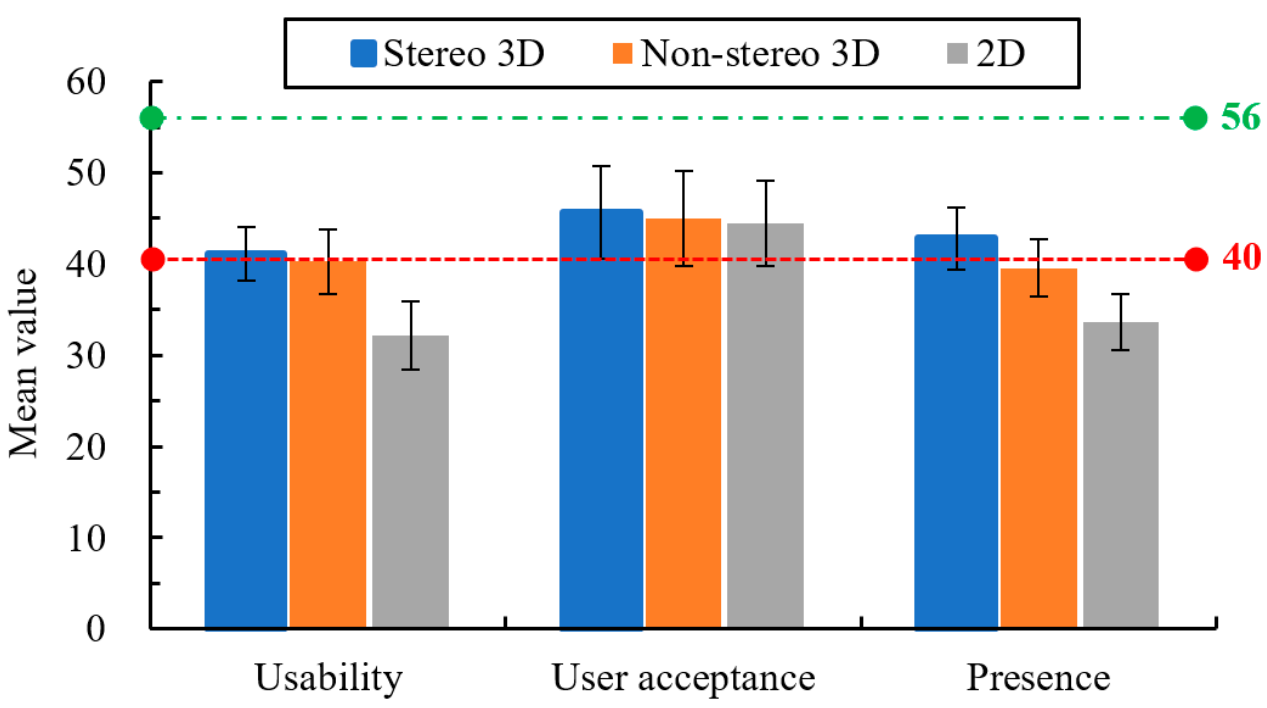

Figure 20. Usability, user acceptance, and presence mean values and standard deviations in different conditions. 
To test the differences among the stereo 3D, nonstereo 3D, and 2D condition, as predicted in hypothesis 2, a one-way analysis of variance (ANOVA) was carried out (Table 5). The homogeneity of variance was tested first, and the sample data all satisfied the requirements of homogeneity $(p>0.05)$. Combined with the mean values (Figure 20), the differences between the conditions were significant regarding usability and presence following the direction predicted $(\mathrm{p}<0.001)$. However, user acceptance was not differentiated by the three conditions.

Table 5. Usability, user acceptance, and presence mean differences based on one-way analysis of variance.

\begin{tabular}{ccccc}
\hline \multirow{2}{*}{ Measure } & \multicolumn{2}{c}{ Homogeneity Test } & \multicolumn{2}{c}{ ANOVA } \\
\cline { 2 - 5 } & $\boldsymbol{L}$ & $\boldsymbol{p}$ & $\boldsymbol{F}$ & $\boldsymbol{p}$ \\
\hline Usability & 0.306 & 0.738 & 31.265 & 0.000 \\
User acceptance & 0.041 & 0.960 & 0.219 & 0.804 \\
Presence & 0.042 & 0.959 & 31.451 & 0.000 \\
\hline
\end{tabular}

In general, Sino-InSpace achieves a highly positive evaluation in usability and presence, which reflects the advantage in practicability and immersion of the simulation. Moreover, a VR setup with $3 \mathrm{D}$ view is more beneficial for potential users in comparison with conventional plane views. However, participants perceived a medium-level of user acceptance, which explains the willingness to adopt the platform. Therefore, further research should be focused on enriching the interactive means and attempting to add more sensory elements (e.g., auditory, gustation, smell, and tactile). Although we looked deeply at the performance by means of questionnaires, there are still limitations associated with this research that affect the ability to generalize the results. In the future, more and various users should be gathered for the evaluation; meanwhile, some objective measuring tasks and methods reflecting the characteristics of the platform will be designed and conducted.

\section{Discussion}

With the development and progress of space technology, outer space is becoming a new living space for human beings. Thus, the expansion of VGEs to VSEs has become imperative. In this study, we independently designed a digital simulation platform for VSEs, known as Sino-InSpace. Based on the analysis of the platform orientation, the details of key technologies were introduced. To meet the needs of multilevel users, multilevel application modes were accordingly designed, each of which had related case studies. These studies demonstrated not only the visual effect but also the quantitative performance in a practical application. The efficiency of the platform can also be verified with the supplementary materials (Video S1). Furthermore, the subjective evaluations with human participants confirmed the value of the platform and the benefits of the VR setup from multiple perspectives.

Sino-InSpace is a product of multidisciplinary integration and has distinctive technical features in comparison with existing platforms. These are as follows: (i) the platform transcends the concept of traditional digital Earth and enriches the paradigm of VGEs. With more frequent interplanetary activities in the future, Sino-InSpace has an advantage over simulation platforms restricted to a single planet in that it supports the integration, expression, and analysis of spatial activity data within the solar system. (ii) It supports multidisciplinary dynamic collaborative deduction. Practitioners from different disciplines (e.g., astronomy, space environment science, geography, etc.) can participate in the platform to conduct scientific simulation experiments throughout secondary development. In contrast, most similar platforms serve a single disciplinary field. (iii) Sino-InSpace considers multilevel users early in its design process and supports applications of direct application, visualization development, and scientific analysis. These modes can support diversified needs, which improves popularization greatly.

The platform is still in the preliminary stage (version 1.0), and the following aspects will be considered in future work. (i) Considering the platform involves multidisciplinary interactions and 
various users, the simulation calculation modules need to be accessed using different operating systems, programming languages, and data interfaces. To address these issues, we will use Service-Oriented Architecture (SOA), register professional modules of different fields with a backstage service center, provide data support simultaneously for multiple stages through an accessible data interface, and improve the simulation efficiency and interoperability. (ii) Based on the concept of the Pan-spatial Information System [82], our platform will be modified to gradually increase the support for indoor (e.g., space station and lunar base) and underground space (e.g., planetary geological survey) in the future. (iii) Based on the concept of geographic scenes, the platform will improve the description for the semantics and relationships of scene elements and constantly enrich the content for scene modeling; in addition, with the aid of VR and AR equipment (e.g., HTC Vive, Oculus Rift, and HoloLens), we will try to use visual and auditory perception methods to enhance the user's ability to understand space environments.

Supplementary Materials: The following are available online at https://pan.baidu.com/s/1BC9EIrSkT7gh8mUMtVUfA, Video S1: Sino-InSpace.

Author Contributions: L.L. and Q.X. conceived and designed the research; L.L., C.L., Q.S., and W.L. developed the Sino-InSpace together. Y.Z. and Y.Z. participated in data collection, application debugging and platform evaluation. L.L. and Y.Z. wrote the paper.

Funding: This research was funded by the National Program on Key Basic Research Project of China (Grant No. 2012CB720000-G), the National Natural Science Foundation of China (Grant Nos. 41701463 and 41371436), and the National Key R\&D Program of China (Grant Nos. 2016YFB0801301 and 2017YFC1200300).

Acknowledgments: We would like to thank all the individuals who contributed in the development of the Sino-InSpace platform including the students who assisted in improving the platform by testing, reporting bugs, and adding functions. We have to extend our heartfelt thanks to all of participants who took part in evaluating the platform. We are also grateful to our users for their patience and suggestions and the reviewers for their helpful comments on this manuscript. We thank Yanxia Cai from NSSC for instruction in the AE8/AP8 model.

Conflicts of Interest: The authors declare no conflict of interest.

\section{Appendix A}

\section{Usability}

Please indicate your response by selecting just ONE of the numbers using the 5-point scale below.

\begin{tabular}{|c|c|c|c|c|}
\hline Almost Never & Some of the Time & About half of the Time & Most of the Time & Almost Always \\
\hline 1 & 2 & 3 & 4 & 5 \\
\hline
\end{tabular}

Is the platform user friendly?

Does this platform provide sufficient content for understanding the solar system?

Does this platform provide precise description for elements in the solar system?

Are you satisfied with the efficiency of data loading?

Are you satisfied with the effect of visualization?

Does this platform provide huge and high-resolution geographical information of planetary surface?

Does this platform improve your cognition of the abstract space environment?

Is it convenient for you to construct a needed scenario?

Does the script meet your needs for deduction?

Assuming you are a space science researcher, would you intend to use this platform for simulation and public education?

\section{User Acceptance}

Please indicate your response by selecting just ONE of the numbers using the 7-point scale below. 


\begin{tabular}{|c|c|c|c|c|c|c|}
\hline $\begin{array}{c}\text { Extremely } \\
\text { Unlikely }\end{array}$ & $\begin{array}{c}\text { Quite } \\
\text { Unlikely }\end{array}$ & $\begin{array}{c}\text { Slightly } \\
\text { Unlikely }\end{array}$ & Neither & $\begin{array}{c}\text { Slightly } \\
\text { Likely }\end{array}$ & Quite Likely & $\begin{array}{c}\text { Extremely } \\
\text { Likely }\end{array}$ \\
\hline 1 & 2 & 3 & 4 & 5 & 6 & 7 \\
\hline
\end{tabular}

My interaction with this platform is clear and understandable.

Learning to operate this platform was easy for me.

I find it easy and fluent to go anywhere I intend to go.

I find it easy to control and switch the observation viewpoint.

I find it flexible to control the simulation time.

The platform enables me to accomplish the addition of environment data quickly.

It is easy for me to remember how to construct a normal scenario.

It is easy for me to learn how to edit a script of deduction.

I find it easy to recover from errors encountered while using the platform.

The platform provides helpful guidance in performing tasks.

\section{Presence}

Please indicate how much you agree or disagree with each of the following statements by circling just ONE of the numbers using the 5-point scale below.

\begin{tabular}{|c|c|c|c|c|}
\hline Strongly Disagree & Disagree & Neutral & Agree & Strongly Agree \\
\hline 1 & 2 & 3 & 4 & 5 \\
\hline
\end{tabular}

To maintain the validity of the copyright owned by the UK Independent Television Commission, 10 items from ITC-SOPI [83] cannot be published in this journal.

\section{Appendix B}

\section{Software Design}

Please answer the following questions by circling the relevant number.

Does this platform provide sufficient functions and interfaces for your projects?
Not at all
1
2
3
4
5
Extremely
sufficient

Does this platform provide the correct functions and interfaces?

Always

wrong

1

23

4

5

Always right

How well do you think the platform performed in fault tolerance?

$\begin{array}{lllllll}\text { Very poor } & 1 & 2 & 3 & 4 & 5 & \text { Very well }\end{array}$

Are the components of platform stable enough for further development?

$\begin{array}{lllllll}\text { Not at all } & 1 & 2 & 3 & 4 & 5 & \begin{array}{c}\text { Extremely } \\ \text { stable }\end{array}\end{array}$

Does adding or modifying a function influence another?
Almost
12
3
4
5
Not at all
Is the coupling between different components strong?
Very low
1
2
3
4
5
Very strong
Is the description of functions and interfaces clear and legible?
Not at all
1
2
3
4
5
Extremely clear and readable

To what extent can the execution efficiency of the platform meet the needs of simulation?
Not at all
1
2
34
5
A lot

How often, on average, does the platform crash or make an error? 


$\begin{array}{cllllll}\begin{array}{c}\text { Almost } \\ \text { always }\end{array} & 1 & 2 & 3 & 4 & 5 & \begin{array}{c}\text { Almost } \\ \text { never }\end{array}\end{array}$

To what extent do you feel that the platform is easy to develop?

$\begin{array}{lllllll}\text { Not at all } & 1 & 2 & 3 & 4 & 5 & \begin{array}{c}\text { Extremely } \\ \text { easy }\end{array}\end{array}$

\section{References}

1. Guo, H.; Dou, C.; Zhang, X.; Han, C.; Yue, X. Earth observation from the manned low earth orbit platforms. ISPRS J. Photogramm. Remote Sens. 2016, 115, 103-118. [CrossRef]

2. Wu, W.R.; Dong, Q.; Jie, D.G. Investigation on the development of deep space exploration. Sci. China Technol. Sci. 2012, 55, 1086-1091. [CrossRef]

3. Ye, P.; Peng, J. Deep space exploration and its prospect in china. Eng. Sci. 2006, 8, 13-18.

4. Zheng, Y.; Ouyang, Z.; Li, C.; Liu, J.; Zou, Y. China's lunar exploration program: Present and future. Planet. Space Sci. 2008, 56, 881-886. [CrossRef]

5. NASA. The Vision for Space Exploration. Available online: https://www.nasa.gov/pdf/55583main_vision_ space_exploration2.pdf (accessed on 1 December 2017).

6. ESA. The Future of European Space Exploration. Available online: http://sci.esa.int/science-e/www/ object $/$ doc.cfm?fobjectid=45512 (accessed on 22 November 2017).

7. Kane, V. Russia's Ambitious Planetary Exploration Goals. Available online: http:/ /www.planetary.org/blogs / guest-blogs/van-kane/20140123-russia-lunar-and-planetary-plans.html (accessed on 1 December 2017).

8. Lin, H.; Chen, M.; Lu, G. Virtual geographic environment: A workspace for computer-aided geographic experiments. Ann. Assoc. Am. GeoGraph. 2013, 103, 465-482. [CrossRef]

9. Steiniger, S.; Hunter, A.J.S. The 2012 free and open source gis software map-A guide to facilitate research, development, and adoption. Comput. Environ. Urban Syst. 2013, 39, 136-150. [CrossRef]

10. Hargitai, H.I. Planetary maps: Visualization and nomenclature. CartoGraph. Int. J. GeoGraph. Inf. Geovis. 2006, 41, 149-165. [CrossRef]

11. USGS. Lunar Web Mapping Application. Available online: https:/ /webgis2.wr.usgs.gov/Lunar_Global_ GIS/ (accessed on 23 September 2017).

12. USGS. Mars Global GIS Mapping Application. Available online: https://webgis2.wr.usgs.gov/Mars_ Global_GIS/ (accessed on 23 September 2017).

13. IAU. Gazetteer of Planetary Nomenclature. Available online: https://planetarynames.wr.usgs.gov/ (accessed on 18 September 2017).

14. Google. Google Earth. Available online: http:/ / earth.google.com/ (accessed on 21 November 2017).

15. NASA. NASA WorldWind. Available online: https://worldwind.arc.nasa.gov/ (accessed on 1 December 2017).

16. ESRI. ArcGIS Earth. Available online: http://www.esri.com/software/arcgis-earth (accessed on 1 December 2017).

17. Guo, H.; Fan, X.; Wang, C. A digital earth prototype system: Deps/cas. Int. J. Digit. Earth 2009, 2, 3-15. [CrossRef]

18. Gong, J.; Chen, J.; Xiang, L.; Xiong, H.; Wu, H.; Wang, Y. Geoglobe:Geo-spatial information sharing platform as open virtual earth. Acta Geod. Cartograph. Sin. 2010, 39, 551-553.

19. Wood, C.A.; Reese, D.D.; Ruberg, L.; Harrison, A.; Lightfritz, C.; Avatrian, L. Moonworld: Implementation of virtual lunar exploration, In Proceedings of the 41st Lunar and Planetary Science Conference, The Woodlands, TX, USA, 1-5 March 2010.

20. Chen, M.; Lin, H.; Wen, Y.; He, L.; Hu, M. Sino-virtualmoon: A 3D web platform using chang'e-1 data for collaborative research. Planet. Space Sci. 2012, 65, 130-136. [CrossRef]

21. Google. Google Mars. Available online: https://www.google.com/mars/ (accessed on 21 November 2017).

22. ASU. JMARS. Available online: https:/ /jmars.asu.edu/ (accessed on 15 August 2017).

23. Huffman, J.; Forsberg, A.; Loomis, A.; Head, J.; Dickson, J.; Fassett, C. Integrating advanced visualization technology into the planetary geoscience workflow. Planet. Space Sci. 2011, 59, 1273-1279. [CrossRef] 
24. Head, J.W.; Dam, A.V.; Fulcomer, S.G.; Forsberg, A.; Rosser, G.; Milkovich, S. Adviser: Immersive scientific visualization applied to mars research and exploration. Photogramm. Eng. Remote Sens. 2005, 71, 1219-1225. [CrossRef]

25. AGI. Systems Tool Kit. Available online: https://www.agi.com/products/stk/ (accessed on 10 October 2017).

26. AGI. Cesium. Available online: https://cesiumjs.org/ (accessed on 10 October 2017).

27. NASA. General Mission Analysis Tool. Available online: https://sourceforge.net/projects/gmat/ (accessed on 1 December 2017).

28. Wood, L. SaVi Satellite Constellation Visualizer. Available online: https://sourceforge.net/projects/savi/ ?source $=$ recommended (accessed on 1 December 2017).

29. Génot, V.; Beigbeder, L.; Popescu, D.; Dufourg, N.; Gangloff, M.; Bouchemit, M.; Caussarieu, S.; Toniutti, J.P.; Durand, J.; Modolo, R. Science data visualization in planetary and heliospheric contexts with 3dview. Planet. Space Sci. 2017, 150, 111-130. [CrossRef]

30. NASA. Cosmographia. Available online: http://naif.jpl.nasa.gov/naif/cosmographia.html (accessed on 1 December 2017).

31. Goodchild, M.F.; Guo, H.; Annoni, A.; Bian, L.; De, B.K.; Campbell, F.; Craglia, M.; Ehlers, M.; Van, G.J.; Jackson, D. Next-generation digital earth. PNAS 2012, 109, 11088-11094. [CrossRef] [PubMed]

32. Xu, B.; Lin, H.; Chiu, L.; Hu, Y.; Zhu, J.; Hu, M.; Cui, W. Collaborative virtual geographic environments: A case study of air pollution simulation. Inf. Sci. 2011, 181, 2231-2246. [CrossRef]

33. Lin, H.; Zhu, J.; Gong, J.; Xu, B.; Qi, H. A grid-based collaborative virtual geographic environment for the planning of silt dam systems. Int. J. GeoGraph. Inf. Sci. 2010, 24, 607-621. [CrossRef]

34. Huang, L.; Gong, J.; Li, W.; Xu, T.; Shen, S.; Liang, J.; Feng, Q.; Zhang, D.; Sun, J. Social force model-based group behavior simulation in virtual geographic environments. ISPRS Int. J. Geo-Inf. 2018, 7, 79. [CrossRef]

35. Lü, G. Geographic analysis-oriented virtual geographic environment: Framework, structure and functions. Sci. China Earth Sci. 2011, 54, 733-743. [CrossRef]

36. Lin, H.; Chen, M. Managing and sharing geographic knowledge in virtual geographic environments (vges). Ann. GIS 2015, 21, 261-263. [CrossRef]

37. Lin, H.; Batty, M.; Jørgensen, S.E.; Fu, B.; Konecny, M.; Voinov, A.; Torrens, P.; Lu, G.; Zhu, A.X.; Wilson, J.P. Virtual environments begin to embrace process-based geographic analysis. Trans. GIS 2015, 19, 493-498. [CrossRef]

38. Chen, M.; Lin, H.; Hu, M.; He, L.; Zhang, C. Real-geographic-scenario-based virtual social environments: Integrating geography with social research. Environ. Plan. B Plan. Des. 2013, 40, 1103-1121. [CrossRef]

39. Chen, M.; Lin, H.; Kolditz, O.; Chen, C. Developing dynamic virtual geographic environments (vges) for geographic research. Environ. Earth Sci. 2015, 74, 6975-6980. [CrossRef]

40. Lin, H.; Chen, M.; Lu, G.; Zhu, Q.; Gong, J.; You, X.; Wen, Y.; Xu, B.; Hu, M. Virtual geographic environments (vges): A new generation of geographic analysis tool. Earth Sci. Rev. 2013, 126, 74-84. [CrossRef]

41. Chen, M.; Lin, H. Virtual geographic environments (vges): Originating from or beyond virtual reality (vr)? Int. J. Digit. Earth 2018, 11, 329-333. [CrossRef]

42. Lü, G.; Yu, Z.; Zhou, L.; Wu, M.; Sheng, Y.; Yuan, L. Data environment construction for virtual geographic environment. Environ. Earth Sci. 2015, 74, 7003-7013. [CrossRef]

43. NASA. PDS: The Planetary Atmospheres Node. Available online: http://pds-atmospheres.nmsu.edu/ (accessed on 1 December 2017).

44. NOAA. Space Weather. Available online: https://ngdc.noaa.gov/stp/spaceweather.html (accessed on 17 December 2017).

45. Picone, J.M.; Hedin, A.E.; Drob, D.P.; Aikin, A.C. Nrlmsise-00 empirical model of the atmosphere: Statistical comparisons and scientific issues. J. Geophys. Res. 2002, 107, 1-16. [CrossRef]

46. Thébault, E.; Finlay, C.C.; Beggan, C.D.; Alken, P.; Aubert, J.; Barrois, O.; Bertrand, F.; Bondar, T.; Boness, A.; Brocco, L. International geomagnetic reference field: The 12th generation. Earth Planets Space 2015, 67, 79. [CrossRef]

47. Bilitza, D.; Altadill, D.; Zhang, Y.; Mertens, C.; Truhlik, V.; Richards, P.; Mckinnell, L.A.; Reinisch, B. The international reference ionosphere 2012-A model of international collaboration. J. Space Weather Space Clim. 2014, 4, 689-721. [CrossRef] 
48. Hog, E.; Fabricius, C.; Makarov, V.V.; Urban, S.; Corbin, T.; Wycoff, G.; Bastian, U.; Schwekendiek, P.; Wicenec, A. The tycho-2 catalogue : Positions, proper motions and two-colour photometry of the 2.5 million brightest stars. Astron. Astrophys. 2000, 355, 27-30.

49. JPL. Jet Propulsion Laboratory Development Ephemeris (JPL DE) 405. Available online: https://en. wikipedia.org/wiki/Jet_Propulsion_Laboratory_Development_Ephemeris (accessed on 17 December 2017).

50. IAU. International Astronomical Union. Available online: https://www.iau.org/ (accessed on 18 September 2017).

51. IERS. International Earth Rotation and Reference Systems Service. Available online: https://www.iers.org/ IERS/EN/Home/home_node.html (accessed on 16 September 2017).

52. IUGG. International Union of Geodesy and Geophysics. Available online: http:/ /www.iugg.org/ (accessed on 16 September 2017).

53. Hohenkerk, C. Standards of fundamental astronomy. Scholarpedia 2011, 6, 11404. [CrossRef]

54. $\mathrm{Xu}, \mathrm{G} . ; \mathrm{Xu}, \mathrm{J}$. Coordinate and time systems. In Orbits: 2nd Order Singularity-Free Solutions, 2nd ed.; Springer: Berlin/Heidelberg, Germany, 2013; ISBN 3642327923.

55. Petit, G.; Luzum, B.; Al, E. IERS Conventions (2010). Available online: https://www.iers. org/SharedDocs/Publikationen/EN/IERS/Publications/tn/TechnNote36/tn36.pdf;jsessionid= 0575758F39B0CFC0E709A864DCB9313C.live1?_blob=publicationFile\&v=1 (accessed on 3 September 2017).

56. Zhang, P.; Xu, C.; Hu, C.; Chen, Y.; Zhao, J. Time scales and time transformations among satellite navigation systems. In China Satellite Navigation Conference (CSNC) 2012 Proceedings; Sun, J., Liu, J., Yang, Y., Fan, S., Eds.; Springer: Guangzhou, China, 2012; pp. 491-502.

57. Chen, M.; Lin, H.; Wen, Y.; He, L.; Hu, M. Construction of a virtual lunar environment platform. Int. J. Digit. Earth 2013, 6, 469-482. [CrossRef]

58. Bo, H.G.; Wu, L.X.; Yu, J.Q.; Yang, Y.Z.; Xie, L. Rapid visualization of global image and dem based on SDOG-ESSG. Int. Arch. Photogramm. Remote Sens. Spat. Inf. Sci. 2013, XL-4/W2, 101-105. [CrossRef]

59. Mahdavi-Amiri, A.; Alderson, T.; Samavati, F. A survey of digital earth. Comput. Graph. 2015, 53, 95-117. [CrossRef]

60. Sperber, M. Quadtree and octree. In Encyclopedia of GIS, 2nd ed.; Shekhar, S., Xiong, H., Zhou, X., Eds.; Springer: Cham, Switzerland, 2017; pp. 1695-1700.

61. Yu, J.; Wu, L.; Zi, G.; Guo, Z. Sdog-based multi-scale 3d modeling and visualization on global lithosphere. Sci. China Earth Sci. 2012, 55, 1012-1020. [CrossRef]

62. Yu, J.; Wu, L. Spatial subdivision and coding of a global three-dimensional grid: Spheoid degenerated-octree grid. In Proceedings of the 2009 IEEE International Geoscience and Remote Sensing Symposium (IGARSS), Cape Town, South Africa, 12-17 July 2010; pp. 361-364. [CrossRef]

63. Lü, G.; Chen, M.; Yuan, L.; Zhou, L.; Wen, Y.; Wu, M.; Hu, B.; Yu, Z.; Yue, S.; Sheng, Y. Geographic scenario: A possible foundation for further development of virtual geographic environments. Int. J. Digit. Earth 2017, 356-368. [CrossRef]

64. Mania, K.; Badariah, S.; Coxon, M.; Watten, P. Cognitive transfer of spatial awareness states from immersive virtual environments to reality. Acm. Trans. Appl. Percept. 2010, 7, 1-14. [CrossRef]

65. IAU. All Named Features for the Moon. Available online: https://planetarynames.wr.usgs.gov/shapefiles/ MOON_nomenclature.zip (accessed on 3 December 2017).

66. Tsyganenko, N.A. Modeling the earth's magnetospheric magnetic field confined within a realistic magnetopause. J. Geophys. Res. 1995, 100, 5599. [CrossRef]

67. Krevelen, R.V.; Poelman, R. A survey of augmented reality technologies, applications and limitations. Int. J. Virtual Reality 2010, 9, 1-20.

68. Gobbetti, E.; Scateni, R. Virtual reality: Past, present and future. Stud. Health Technol. Inform. 1998, 58, 3-20. [PubMed]

69. Schnabel; Aurel, M.; Wang, X.; Seichter, H.; Kvan, T. Touching the untouchables: Virtual-, augmentedand reality. In Proceedings of the Association for Computer-Aided Architectural Design Research in Asia (CAADRIA 2008), Chaiang Mai, Thailand, 9-12 April 2008.

70. Steuer, J.S. Defining virtual reality: Dimensions determining telepresence. J. Commun. 1992, 42, 73-93. [CrossRef]

71. Wang, X.; Kim, M.J.; Love, P.E.D.; Kang, S.C. Augmented reality in built environment: Classification and implications for future research. Autom. Constr. 2013, 32, 1-13. [CrossRef] 
72. Baidu. ECharts. Available online: https://ecomfe.github.io/echarts-doc/public/en/index.html (accessed on 4 February 2018).

73. Jordan, C.E. Nasa radiation belt models ap-8 and ae-8. J. Polit. 1989, 51, 212-214.

74. Bai, X.Z.; Chen, L.; Zhang, Y.; Tang, G.J. Survey on collision assessment and warning techniques for space object. J. Astronaut. 2013, 8, 1027-1039.

75. USGS. Mars Colorized Viking Mosaic. Available online: https://planetarymaps.usgs.gov/mosaic/Mars_ Viking_MDIM21_ClrMosaic_global_232m.tif (accessed on 3 December 2017).

76. Smith, D.; Neumann, G.; Arvidson, R.E.; Guinness, E.A.; Slavney, S. MGS-M-MOLA-5-MEGDR-L3-V1.0. Available online: https:/ / pds.nasa.gov/ds-view / pds/viewProfile.jsp?dsid=MGS-M-MOLA-5-MEGDRL3-V1.0 (accessed on 4 February 2018).

77. Yoon, S.Y.; Laffey, J.; Oh, H. Understanding usability and user experience of web-based 3D graphics technology. Int. J. Hum. Comput. Interact. 2008, 24, 288-306. [CrossRef]

78. Davis, F.D. Perceived usefulness, perceived ease of use, and user acceptance of information technology. Mis. Q. 1989, 13, 319-340. [CrossRef]

79. Ijsselsteijn, W. Elements of a multi-level theory of presence: Phenomenology, mental processing and neural correlates. In Proceedings of the Presence, Porto, Portugal, 9-11 October 2002; pp. 245-259.

80. Habra, N.; Abran, A.; Lopez, M.; Sellami, A. A framework for the design and verification of software measurement methods. J. Syst. Softw. 2008, 81, 633-648. [CrossRef]

81. Cronbach, L.J. Coefficient alpha and the internal structure of tests. Psychometrika 1951, 16, 297-334. [CrossRef]

82. Hua, Y. The core problems and key technologies of pan-spatial information system. J. Geomat. Sci. Technol. 2016, 33, 331-335.

83. Lessiter, J.; Freeman, J.; Keogh, E.; Davidoff, J. A cross-media presence questionnaire: The itc-sense of presence inventory. Presence 2014, 10, 282-297. [CrossRef]

(C) 2018 by the authors. Licensee MDPI, Basel, Switzerland. This article is an open access article distributed under the terms and conditions of the Creative Commons Attribution (CC BY) license (http:/ / creativecommons.org/licenses/by/4.0/). 